\title{
Bargaining with Incomplete Information
}

\author{
Shaheen S. Fatima and Michael Wooldridge \\ ( $\{$ s.s.fatima, m.j.wooldridge\}@csc.liv.ac.uk) \\ Department of Computer Science, University of Liverpool, Liverpool L69 7ZF, \\ $U . K$. \\ Nicholas R. Jennings (nrj@ecs.soton.ac.uk) \\ Department of Electronics and Computer Science, University of Southampton, \\ Southampton SO17 1BJ, U.K.
}

\begin{abstract}
This paper analyses the process and outcomes of competitive bilateral negotiation for a model based on negotiation decision functions. Each agent has time constraints in the form of a deadline and a discounting factor. The importance of information possessed by participants is highlighted by exploring all possible incomplete information scenarios - both symmetric and asymmetric. In particular, we examine a range of negotiation scenarios in which the amount of information that agents have about their opponent's parameters is systematically varied. For each scenario, we determine the equilibrium solution and study its properties. The main results of our study are as follows. Firstly, in some scenarios agreement takes place at the earlier deadline, while in others it takes place near the beginning of negotiation. Secondly, in some scenarios the price surplus is split equally between the agents while in others the entire price surplus goes to a single agent. Thirdly, for each possible scenario, the equilibrium outcome possesses the properties of uniqueness and symmetry - although it is not always Pareto optimal. Finally, we also show the relative impacts of the opponent's parameters on the bargaining outcome.
\end{abstract}

Keywords: Negotiation, Game Theory, Incomplete Information

\section{Introduction}

Negotiation is a means for agents to communicate and compromise to reach mutually beneficial agreements. In such situations, the agents have a common interest in cooperating, but have conflicting interests over exactly how to cooperate. Put differently, the agents can mutually benefit from reaching agreement on an outcome from a set of possible outcomes, but have conflicting interests over the outcome that they prefer. The main problem that confronts agents in such a situation is to decide how to cooperate before they actually enact the cooperation, and obtain the associated benefits. On the one hand, each agent would like to reach some agreement rather than disagree and not reach any agreement. But, on the other hand, each agent would like to reach an agreement that is as favourable to it as possible.

The negotiation process has long been modelled using the tools of game theory, and these are now being used extensively in the de-

(C) 2003 Kluwer Academic Publishers. Printed in the Netherlands. 
velopment of software agents for automated negotiation (Sandholm, 2000; Jennings et al., 2001; Kraus, 2001; Lomuscio et al., 2003). In such encounters, each agent has to make decisions about generating offers and counter-offers in such a way that its own utility from the final agreement is maximized. An essential input to this decision making process is information (Young, 1975); here defined as the knowledge about all factors which affect the ability of an individual to make choices in any given situation. For example, in bargaining between a buyer and a seller, information includes what an agent knows about its own parameters (like its reservation price or its preferences over possible outcomes), and also what it knows about its opponent (like the opponent's reservation price or the opponent's preferences over possible outcomes).

Game theoretic models for bargaining can be divided into two types: those that deal with complete information and those that deal with incomplete information. In the former setting, agents know each other's parameters as well as their own (Nash, 1950). In the latter setting, agents lack information about some specific parameters. For instance, there could be uncertainty over the players' discounting factors (Rubinstein, 1985), reservation prices (Fudenberg et al., 1985), or deadlines (Sandholm and Vulkan, 1999). These models study the strategic behaviour of agents when there is information uncertainty. Our objective here is not to address the issue of uncertainty per se, but rather to analyse the impact on the negotiation outcome of knowing various pieces of information about the opponent's parameters. We therefore explore a range of negotiation scenarios by varying the degree of information an agent has about its opponent.

To provide a concrete setting for our study, we consider negotiation between a buyer and a seller over the price of a good or service. Negotiation needs to be completed by a specified time, which is likely to be different for the different parties. Apart from the agents' respective deadlines, the time at which agreement is reached can effect the agents in different ways (Fatima et al., 2002c). An agent can gain utility with time and have the incentive to reach a late agreement (within its deadline). In such a case it is said to be a patient player. The other possibility is that it can lose utility with time, and try to reach an early agreement. It is then said to be an impatient $^{1}$ player. As we will show, this disposition and the actual deadline itself strongly influence the negotiation outcome. Apart from this, the agents' reservation limits

\footnotetext{
${ }^{1}$ (Kraus, 2001) provides examples of scenarios in which agents gain and lose utility with time.
} 
also influence the outcome. We therefore study the effect of all these parameters on the equilibrium solution.

In more detail, we analyse the mutual strategic behaviour of agents for a particular negotiation model based on negotiation decision functions (see Section 2 for details). This analysis is done for a complete range of symmetric and asymmetric information scenarios. In (Fatima et al., 2002a) we examined the influence of information on the negotiation equilibrium for symmetric information scenarios. Here we extend this to asymmetric information scenarios. In each of these situations we determine equilibrium strategies and study how the information state of agents influences the division of gains from trade between agents. We say that an agent has more/less bargaining power than its opponent if this division is more/less favourable to it than to its opponent. In addition, we study the properties of the equilibrium solution.

This paper advances the state of the art in that such an analysis has not previously been undertaken. We believe this analysis is particularly useful for constructing software agents for electronic commerce for the following reasons. Firstly, it uses simple functions for generating offers and counter-offers that have shown to be practical in e-commerce scenarios (Jennings et al., 2000). Secondly, it takes the time constraints of bargainers into consideration, both in the form of deadlines and discounting factors. Thirdly, it provides a comprehensive study of all possible incomplete information scenarios that include both symmetric and asymmetric information. Time and information are the two important aspects of negotiation in this domain, and player strategies and the outcomes depend on both these factors (Rubinstein, 1985). Fourthly, the equilibrium solution generated by our model is robust, i.e., has the property of uniqueness and symmetry for each possible scenario. However, the bargaining outcome is not always Pareto optimal, since agents bargain with incomplete information. Finally, we also show the relative impacts of the opponent's parameters on the negotiation outcome.

The remainder of the paper is structured as follows. Section 2 describes our negotiation model. Section 3 determines the optimal and equilibrium strategies for symmetric information scenarios. The analysis for asymmetric information scenarios is carried out in Section 4. In Section 5 we compare the influence of negotiation parameters on the bargaining power of agents. Section 6 studies the properties of equilibrium solutions and Section 7 discusses related work. Finally in Section 8 we present our conclusions. Appendix A provides a summary of the notation employed throughout the paper. Appendix B summarizes the equilibrium outcomes for both symmetric and asymmetric information scenarios. 


\section{The Negotiation Model}

We use an alternating offers protocol for our study (Rubinstein, 1982). Let $b$ denote the buyer, $s$ the seller and let $\left[I P^{a}, R P^{a}\right]$ denote the range of values for price that is acceptable to agent $a$, where $a \in\{b, s\}$. Let $\hat{a}$ denote agent $a$ 's opponent. A price that is acceptable to both $b$ and $s$, i.e., the zone of agreement $(Z)$, is the interval $\left[R P^{s}, R P^{b}\right]$. The difference between $R P^{b}$ and $R P^{s}$ is called the price-surplus. $T^{a}$ denotes agent $a$ 's deadline. Let $p_{b \rightarrow s}^{t}$ denote the price offered by $b$ to $s$ at time $t$. Negotiation starts when the first offer is made. When an agent, say $s$, receives an offer at time $t$, i.e., $p_{b \rightarrow s}^{t}$, it rates the offer using its utility function $U^{s}$ where $U^{s}:$ Offers $\times$ Times $\rightarrow \mathbb{R}$. The set Offers is a set of values for price and the set Times is the set of positive integers. If $U^{s}\left(p_{b \rightarrow s}^{t}, t\right)$ is greater than the utility of the counter-offer agent $s$ is ready to send at time $t^{\prime}$, i.e., $p_{s \rightarrow b}^{t^{\prime}}$ with $t^{\prime}>t$, then agent $s$ accepts. Otherwise $s$ makes a counter-offer unless its deadline has passed. So the action that agent $s$ takes at time $t^{\prime}$ is defined as:

$A^{s}\left(t^{\prime}, p_{b \rightarrow s}^{t}\right)= \begin{cases}\text { Quit } & \text { if } t^{\prime}>T^{s} \text { where } T^{s} \text { is the seller's deadline } \\ \text { Accept } & \text { if } U^{s} \text { from } p_{b \rightarrow s}^{t} \geq U^{s} \text { from counter-offer } p_{s \rightarrow b}^{t^{\prime}} \\ p_{s \rightarrow b}^{t^{\prime}} & \text { otherwise. }\end{cases}$

The action for $b$ can be defined analogously. The process of making offers and counter-offers continues until either an agreement is reached, or one of the two deadlines is reached. If the deadline $T^{a}$ is reached without an agreement taking place, then agent $a$ quits and negotiation ends in a conflict.

Since both agents have a deadline, we assume that they use a time dependent function (i.e., linear, Boulware or Conceder (Faratin et al., 1998)), for generating offers. These tactics vary the price depending on the remaining negotiation time, modelled as the above defined constant $T^{a}$. In these functions, the predominant factor used to decide which value to offer next is time $t$. The initial offer is a point in the interval $\left[I P^{a}, R P^{a}\right]$. Agents define a constant $k^{a}$ that, when multiplied by the size of the interval, determines the price to be offered in the first proposal by $a$. The offer made by $a$ at time $t\left(0<t \leq T^{a}\right)$ is defined in terms of the negotiation decision function (NDF), $f^{a}$, as follows:

$$
p_{a \rightarrow \hat{a}}^{t}= \begin{cases}I P^{b}+f^{b}(t)\left(R P^{b}-I P^{b}\right) & \text { for } b \\ R P^{s}+\left(1-f^{s}(t)\right)\left(I P^{s}-R P^{s}\right) & \text { for } s .\end{cases}
$$

A wide range of functions can be defined by varying the way in which $f^{a}(t)$ is computed (see (Faratin et al., 1998) for more details). However, functions must ensure that $0 \leq f^{a}(t) \leq 1, f^{a}(0)=k^{a}$ and 


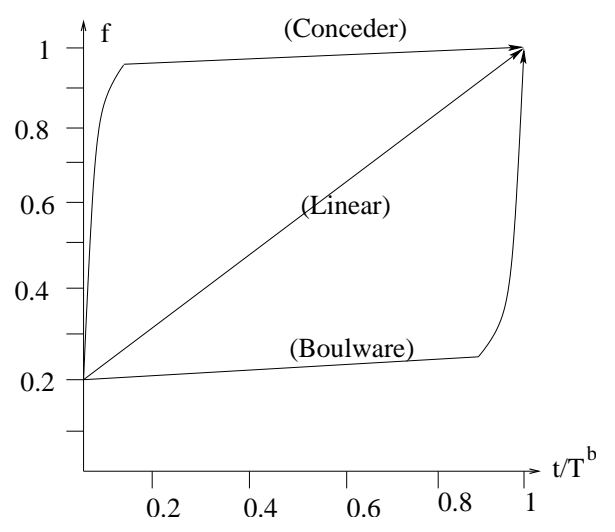

Figure 1. Negotiation decision functions for the buyer

$f^{a}\left(T^{a}\right)=1$. That is, the offer will always be between the value range, at the beginning it will give the initial constant and when the deadline is reached it will offer the reservation value. Function $f^{a}(t)$ is defined as follows:

$$
f^{a}(t)=k^{a}+\left(1-k^{a}\right)\left(\frac{\min \left(t, T^{a}\right)}{T^{a}}\right)^{\frac{1}{\psi}}
$$

An infinite number of functions can be defined for different values of $\psi$. However, three extreme sets show clearly different patterns of behaviour (Raiffa, 1982; Pruitt, 1981) (see Figure 1 ).

1. Boulware $(B)$. For this function, $\psi<1$ and the initial offer is maintained until time is almost exhausted, when the agent concedes up to its reservation value.

2. Conceder $(C)$. For this function, $\psi>1$ and the agent goes to its reservation value very quickly.

3. Linear $(L)$. For $\psi=1$, price is increased linearly.

As it is important for both the agents to reach an agreement before their respective deadlines, the key factor in the generation of offers and counter-offers is the remaining time for negotiation. An agent's strategy is therefore defined in terms of time and not in terms of the history of negotiation. This is explained below. The value of a counter-offer depends on the initial price $(I P)$ at which the agent starts negotiation, the final price $(F P)$ beyond which it does not concede, the time at which $F P$ is offered and $\psi$. 
DEFINITION 1. An agent a's strategy $\left(S^{a}\right)$ is a 4-tuple

$$
\left\langle I P^{a}, F P^{a}, t^{a}, N D F^{a}\right\rangle,
$$

where $I P^{a}$ denotes the agent's initial price, $F P^{a}$ the final price beyond which it does not concede, $t^{a}$ the time at which it offers $F P^{a}$ and the last element denotes its NDF, (i.e., $\left.\psi^{a}\right)$.

DEFINITION 2. The negotiation outcome $(O)$ is an element of the set $\{(p, t), \mathcal{C}\}$, where $(p, t)$ denotes the price and time at which agreement is reached and $\mathcal{C}$ denotes the conflict ${ }^{2}$ outcome.

As an illustration, when $b$ 's strategy is $\left\langle I P^{b}, R P^{b}, T^{s}, B\right\rangle$ and $s$ 's strategy is $\left\langle I P^{s}, R P^{s}, T^{s}, B\right\rangle$, the outcome $\left(O_{1}\right)$ that results is shown in Figure 2(a). Note that each agent offers its final price at the earlier deadline, i.e., $T^{s}$. As shown in the figure, agreement is reached at a price $R P^{s}+\frac{\text { price-surplus }}{2}$ and at a time close to $T^{s}$. Similarly when the NDF in both strategies is replaced with $C$, agreement $\left(\mathrm{O}_{2}\right)$ is reached at the same price but near the beginning of negotiation. Figure 2(b) illustrates a negotiation conflict. The strategies for $b$ and $s$ are $\left\langle I P^{b}, R P^{b}, T^{b}, B\right\rangle$ and $\left\langle R P^{s}, R P^{s}, T^{s}, B\right\rangle$ respectively and $T^{s}<T^{b}$. As agents have unequal deadlines and both agents use the $B$ function, the strategies do not converge and result in a conflict. In general, agents can avoid conflict by using a strategy that offers a mutually acceptable price (i.e., within $Z$ ) by a mutually acceptable time (the earlier deadline). As we will show in the following sections, the optimal strategies that we determine always result in an agreement (see Appendix B for a summary of the outcomes).

Agents' utilities are defined with the following two von NeumannMorgenstern utility functions (Keeney and Raiffa, 1976) that incorporate the effects of discounting and bargaining costs:

$$
U^{a}(p, t)=U_{p}^{a}(p) U_{t}^{a}(t) \quad \text { where } \quad a \in\{b, s\} \text { and } \quad t<T^{a}
$$

$U_{p}^{b}$ is a decreasing function of price and $U_{p}^{s}$ is an increasing function of price. For an agent, $U_{t}^{a}$ increases with time if its discounting factor $\delta^{a}>1$. Consequently, the agent gains utility with time and has an incentive to reach a late agreement. But if $U_{t}^{a}$ decreases with time (i.e., $\left.\delta^{a}<1\right)$ then the agent loses on time and has an incentive to reach an early agreement. Agents are said to have similar time preferences if both gain on time or both lose on time; otherwise they have conflicting time preferences. Let $U^{a}(\mathcal{C})$ denote agent $a$ 's utility from conflict.

\footnotetext{
${ }^{2}$ An agent's optimal strategy should avoid the conflict outcome since an agreement always gives a better utility than conflict. Conflict is the worst outcome.
} 

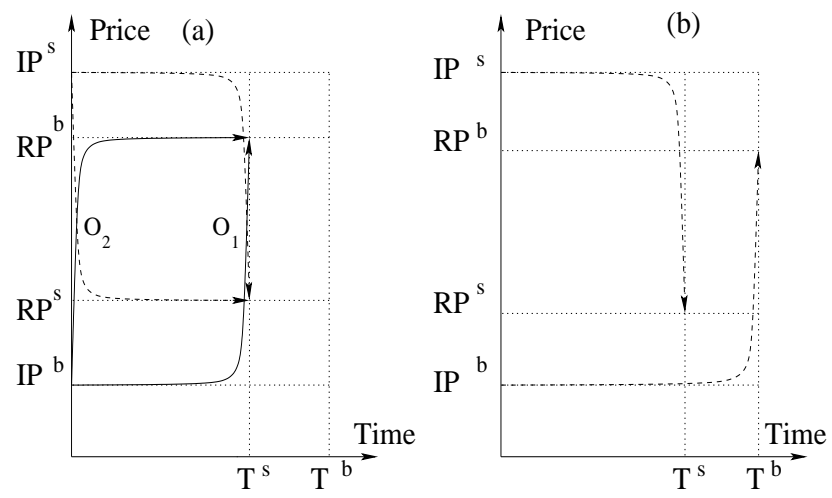

Figure 2. Illustration of agreement and negotiation conflict

Each agent's utility from agreement is always higher than its conflict utility, i.e., $U^{a}(p, t)>U^{a}(\mathcal{C})$ where $p$ is any price within $Z$, the zone of agreement, and $t \leq T^{a}$. Conflict is the worst outcome for both $b$ and $s$.

\section{Equilibrium Outcomes for Symmetric Information Scenarios}

Each agent has a reservation limit, a deadline and a discounting factor. Thus $b$ and $s$ each have three parameters denoted $\left\langle R P^{b}, T^{b}, \delta^{b}\right\rangle$ and $\left\langle R P^{s}, T^{s}, \delta^{s}\right\rangle$ respectively. The outcome of negotiation depends on all these six parameters. The information state of an agent is the information it has about the negotiation parameters. An agent's own parameters are known to it, but the information it has about the opponent's parameters varies.

DEFINITION 3. The information state $I^{a}$ of an agent $a$ is an ordered pair. The first element, denoted $F^{a}$, is a 3-tuple containing its own parameters. This forms the fixed part of $I^{a}$. The second element, denoted $V^{a}$, is an n-tuple containing information about its opponent's parameters where $n$ varies between zero and three. This forms the variable part of $I^{a}$. Thus

$$
I^{a}=\left\langle F^{a}, V^{a}\right\rangle, F^{b}=\left\langle R P^{b}, T^{b}, \delta^{b}\right\rangle, \text { and } F^{s}=\left\langle R P^{s}, T^{s}, \delta^{s}\right\rangle
$$

In the following subsections we vary $n$ between zero and three. For each value of $n$, we determine $b$ 's optimal strategy $S^{b}$ on the basis of $I^{b}$ and $s$ 's optimal strategy $S^{s}$ on the basis of $I^{s}$ where $b$ and $s$ are von Neuman and Morgenstern expected utility maximizers. As described in the 


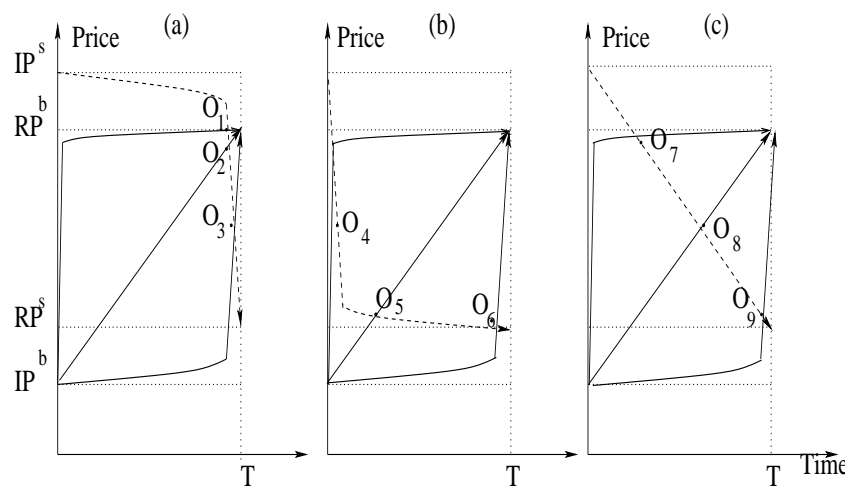

Figure 3. Possible strategies and associated outcomes for $V^{a}=\left\langle T^{\hat{a}}\right\rangle$.

previous section, strategy is a four tuple $S^{a}=\left\langle I P^{a}, F P^{a}, t^{a}, N D F^{a}\right\rangle$. This is optimal if the four elements not only satisfy the constraint of avoiding conflict but also result in agreement at the maximum possible utility. For each agent $a$, we first determine (on the basis of $I^{a}$ ) the four elements that form the optimal strategy $S^{a}$. We then prove that the mutual strategic behaviour of agents where both use their respective optimal strategies form sequential equilibrium points (Osborne and Rubinstein, 1994; Kreps and Wilson, 1982).

The information states of the players can be symmetric or asymmetric. In the symmetric information scenarios, the buyer and the seller have equal information about each other (i.e., they have information on the same parameter(s) about each other). On the other hand, information is said to be asymmetric if the players have information on different parameters about each other (i.e., they have unequal information about each other). This section analyses the symmetric information scenarios and Section 4 deals with the asymmetric case.

\section{1. $V^{a}$ CONTAins A SINGLE ELEMENT}

This covers the cases where $V^{a}$ contains the opponent's deadline, reservation price or discounting factor. The optimal strategy determination for an agent $a$ when it has no information about its opponent's parameters (i.e., $V^{a}=\langle\rangle$ ) is described under the item for $V^{a}=\left\langle\delta^{\hat{a}}\right\rangle$ since both cases have the same optimal strategy.

The following analysis is carried out from the perspective of the buyer. The analysis for the seller can be carried out analogously. 
1. Deadline. When agents know each others' deadline, the information states are $V^{b}=\left\langle T^{s}\right\rangle$ and $V^{s}=\left\langle T^{b}\right\rangle$.

Optimal strategies. In the absence of $R P^{s}, b$ can ensure convergence by making $I P=I P^{b}$ (a very low price that lies outside $Z$ ), $F P^{b}=R P^{b}$, and offering $F P^{b}$ before the earlier deadline. Thus the third element of the strategy becomes $T^{s}$ if $\left(T^{s}<T^{b}\right)$ and $T^{b}$ if $\left(T^{b}<T^{s}\right)$. Given this, the last element, i.e., the NDF, needs to be determined so as to optimise the time of agreement. Figure 3 depicts the negotiation outcome for each of the three NDFs. $T$ denotes the earlier deadline. The dashed lines indicate $s$ 's strategy and the solid lines indicate $b$ 's strategy. Note that the actual values of $I P$ and FP in $s$ 's strategy are not known to $b$ although $b$ knows that $I P^{s}$ is some value greater than $R P^{b}$, and $F P^{s}$ is some value less than $R P^{b}$ and greater than $I P^{b}$. Out of the three NDFs we need to determine the one that always gives $b$ the best possible utility. Agent $b$ can have two possible attitudes towards time. It can gain utility with time and have an incentive to reach a late agreement, or it can lose utility with time and have an incentive to reach an early agreement. Consider the case where $b$ gains on time. If $s$ uses the Boulware NDF, then as seen in Figure 3(a) the outcome can be $O_{1}, O_{2}$, or $O_{3}$ depending on $b$ 's strategy. Of these three, $\mathrm{O}_{3}$ results in agreement at the lowest price and the latest time. Thus if $s$ uses the Boulware NDF, it is best for $b$ to also use the Boulware NDF. Similarly if $s$ uses the Conceder (or Linear) NDF, (see Figures 3(b) and 3(c)) the most favourable outcome to $b$ is $O_{6}$ (or $O_{9}$ ) generated by the Boulware NDF. Thus if $b$ gains on time, irrespective of $s$ 's strategy, it is best for it to always use the Boulware NDF. Agent $b$ 's optimal strategy is therefore $S^{b}=\left\langle I P^{b}, R P^{b}, T, B\right\rangle$. Consider the other possibility where $b$ loses utility on time. Here we consider scenarios where $Z$ is small and $T$ is large. So the gain in utility on time from $O_{5}$ to $O_{4}$ (and $O_{6}$ to $O_{5}$ ) outweighs the loss in utility from price. In other words, agents always try to minimize the time of agreement as long as the price is within the zone of agreement. As shown in Figures 3(a), $3(\mathrm{~b})$, and $3(\mathrm{c})$, irrespective of $s$ 's strategy, $b$ can minimize the time of agreement by using the Conceder NDF. Agent $b$ 's optimal strategy therefore becomes $S^{b}=\left\langle I P^{b}, R P^{b}, T, C\right\rangle$. Analogously, $S^{s}=\left\langle I P^{s}, R P^{s}, T, B / C\right\rangle$, where $I P^{s}$ is some high price outside $Z$ and the last element in $S^{s}$ is $B$ if $s$ gains on time and $C$ if it loses on time.

Since an agent's optimal strategy does not depend on its opponent's strategy, neither agent has the incentive to deviate from it 


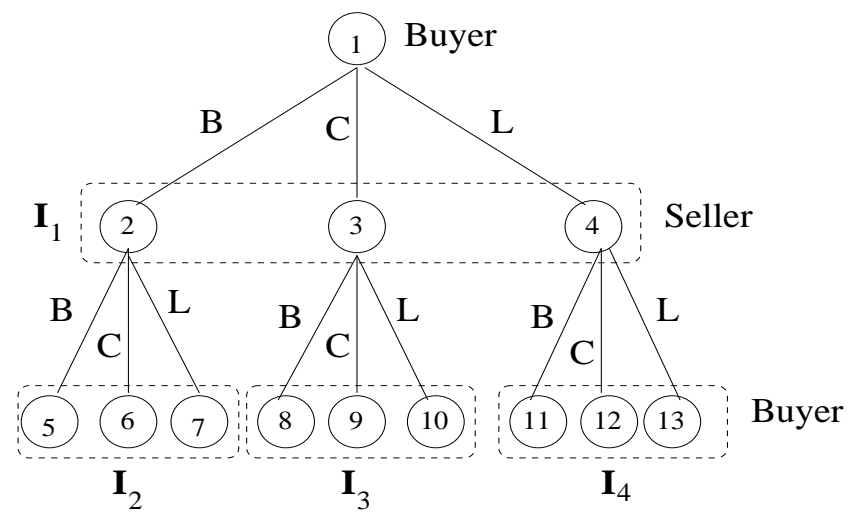

Figure 4. Extensive form of the negotiation game

at any point during negotiation. We now prove that this mutual strategic behaviour of agents forms a sequential equilibrium. As agents do not have information about their opponent's strategy or utility, negotiation can be considered as a game $\mathbf{G}$ of alternating offers and incomplete information. For games of alternating offers, a strategy profile and belief system pair is a sequential equilibrium of an extensive game if it is sequentially rational and consistent (Kreps and Wilson, 1982; Osborne and Rubinstein, 1994). A system of beliefs $\mu$ in $\mathbf{G}$ is a specification of a probability $x \in[0,1]$ for each decision node $x$ in $\mathbf{G}$ such that

$$
\sum_{x \in \mathbf{I}} \mu(x)=1
$$

for all information sets $\mathbf{I}$. In other words, $\mu$ represents the agent's beliefs about the history of negotiation. The player's strategies satisfy sequential rationality if for each information set of each player $a$, the strategy of player $a$ is a best response to the other player's strategies, given $a$ 's beliefs at that information set. The requirement for $\mu$ to be consistent with the strategy profile is as follows. Even at an information set that is not reached, if all players adhere to their strategies, it is required that a player's belief be derived from some strategy profile using Bayes' rule.

THEOREM 1. The strategy profile $S^{b} \times S^{s}$ forms a sequential equilibrium of the game $\boldsymbol{G}$.

Proof. The first three levels of the extensive form for this game (G) are shown in Figure 4. At node 1, one of the players, say b, 
starts negotiation using its optimal strategy $S^{b}$. After the first offer, play reaches node 2. At this level, it is player s's turn to make a decision. $\boldsymbol{I}_{1}$ becomes the information set for $s$ since it is unaware of the strategy used by $b$ and hence does not know which of the three nodes 2, 3 or 4, play has reached. However, irrespective of exactly which node play reaches at this level (i.e., irrespective of s's belief about the history of negotiation), the dominant strategy for $s$ is $S^{s}$. Play now reaches node 5 (since both agents use B) at which $b$ makes a move. At this point $b$ does not know exactly which node the play is at, but it knows that its information set $\boldsymbol{I}_{2}$ is reached with probability 1 (probability of reaching other decision nodes at this level is 0). The dominant strategy for $b$ at this information set (and at all others) is $S^{b}$. Thus at every information set at which it is $b$ 's turn to move, its optimal strategy is $S^{b}$ and at every information set at which it is s's turn to make a move, its optimal strategy is $S^{s}$. The strategy profile $S^{b} \times S^{s}$ therefore satisfies the requirements for sequential rationality. Furthermore, at every information set the optimal strategies are also dominant strategies. This makes the strategy profile $S^{b} \times S^{s}$ a sequential equilibrium irrespective of the agents' beliefs about the history of negotiation.

COROLLARY 1. The equilibrium profile $S^{b} \times S^{s}$ is unique.

Proof. This is a direct consequence of the above proof. As the optimal strategies for both agents are dominant strategies at each of their information sets, there does not exist any other equilibrium (neither a pure nor a mixed strategy) where an agent uses a strategy other than its optimal strategy. The equilibrium solution is therefore unique.

The equilibrium outcomes for $V^{a}=\left\langle T^{\hat{a}}\right\rangle$ in the four possible negotiation scenarios are listed in Table I. The proof of Theorem 1 can be used to show the existence of a unique equilibrium in all the following scenarios.

2. Reservation price $\left(R P^{a}\right)$. Here $V^{b}=\left\langle R P^{s}\right\rangle$ and $V^{s}=\left\langle R P^{b}\right\rangle$. In the absence of $T^{s}$, agent $b$ can ensure convergence if it starts making offers at $R P^{s}$ and reaches $R P^{b}$ by $T^{b}$. Using the same analysis as for $V^{a}=\left\langle T^{\hat{a}}\right\rangle$, we get $S^{b}=\left\langle R P^{s}, R P^{b}, T^{b}, B / C\right\rangle$. The last element is $B$ if $b$ is patient and $C$ if it is impatient. Similarly the optimal strategy for $s$ becomes $S^{s}=\left\langle R P^{b}, R P^{s}, T^{s}, B / C\right\rangle$. The proof of Theorem 1 can also be used to show that a unique equilibrium exists at $S^{b} \times S^{s}$ in this case. The equilibrium outcomes for $V^{a}=\left\langle R P^{\hat{a}}\right\rangle$ are also listed in Table I of Appendix B. 


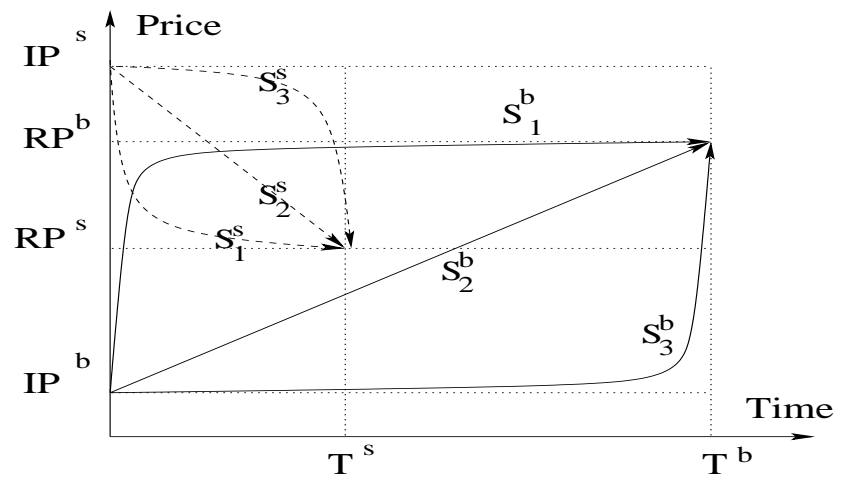

Figure 5. Possible strategies and associated outcomes for $V^{a}=\delta^{\hat{a}}$.

3. Discounting factor $\left(\delta^{a}\right)$. Here $V^{b}=\left\langle\delta^{s}\right\rangle$ and $V^{s}=\left\langle\delta^{b}\right\rangle$. In the absence of any other information about the opponent, the strategies available to the agents are $S^{b}=\left\langle I P^{b}, R P^{b}, T^{b}, B / C\right\rangle$ and $S^{s}=$ $\left\langle I P^{s}, R P^{s}, T^{s}, B / C\right\rangle$. Consider the optimal strategy determination for the buyer. Figure 5 illustrates the case where $T^{s}<T^{b}$. Assume that $\delta^{s}<1$. In this scenario, the buyer can ensure an agreement only by using the Conceder $\operatorname{NDF}\left(S_{1}^{b}\right)$. Any other strategy (i.e., Linear $\left(S_{2}^{b}\right)$ or Boulware $\left.\left(S_{3}^{b}\right)\right)$ can result in a conflict irrespective of the seller's strategy (i.e., $S_{1}^{s}, S_{2}^{s}$, or $S_{3}^{s}$ ). In other words, the buyer knows that $\delta^{s}<1$ and can infer that $s$ will use a Conceder NDF to reach an early agreement. But since $b$ does not know the seller's deadline $\left(T^{s}\right)$ or its reservation price $\left(R P^{s}\right), b$ can ensure convergence of strategies only by using the Conceder NDF. Thus the buyer's optimal strategy is $S^{b}=\left\langle I P^{b}, R P^{b}, T^{b}, C\right\rangle$ and it is independent of $\delta^{s}$. In the same way it can be seen that $b$ 's optimal strategy is $S^{b}=\left\langle I P^{b}, R P^{b}, T^{b}, C\right\rangle$ if $T^{b}<T^{s}$. So irrespective of the relationship between agent deadlines, $b$ 's optimal strategy remains $S^{b}=\left\langle I P^{b}, R P^{b}, T^{b}, C\right\rangle$. Analogously, the seller's optimal strategy becomes $S^{s}=\left\langle I P^{s}, R P^{s}, T^{s}, C\right\rangle$. The strategy profile $S^{b} \times S^{s}=$ $\left\langle I P^{b}, R P^{b}, T^{b}, C\right\rangle \times\left\langle I P^{s}, R P^{s}, T^{s}, C\right\rangle$ forms an equilibrium since only this profile guarantees an agreement. The proof of this is the same as that for $\left(V^{a}=\left\langle T^{\hat{a}}\right\rangle\right)$. See Table I for the corresponding outcomes.

We now turn to the case where $V^{a}=\langle\rangle$. In the preceding paragraph it was shown that an agent's optimal strategy does not depend on $\delta^{\hat{a}}$. Adding $\delta^{\hat{a}}$ to $V^{a}$ or deleting $\delta^{\hat{a}}$ from $V^{a}$ does not alter $a$ 's 


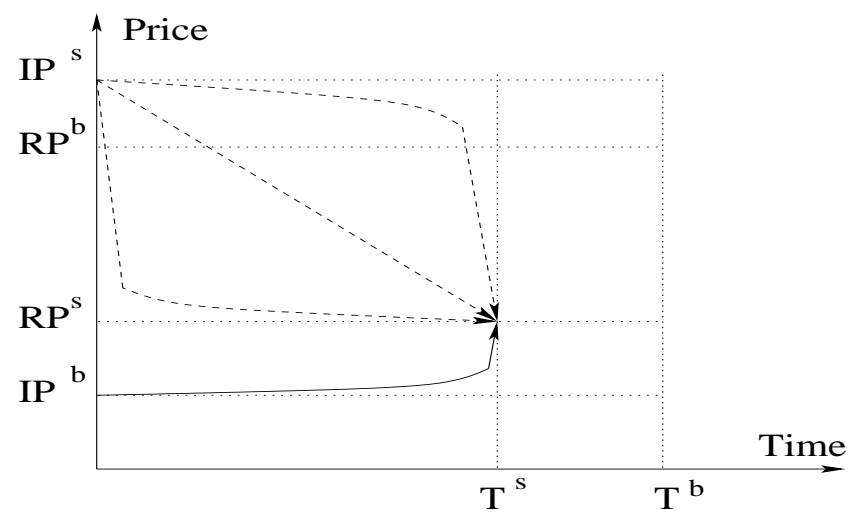

Figure 6. Possible seller strategies for $V^{a}=\left\langle T^{\hat{a}}, R P^{\hat{a}}\right\rangle$

optimal strategy. The agents' optimal strategies for $V^{a}=\langle\rangle$ are therefore the same as those for $V^{a}=\left\langle\delta^{\hat{a}}\right\rangle$.

\section{2. $V^{a}$ CONTAins TWO ELEMENTS}

We now consider the effect of different parameter pairs on the negotiation equilibrium.

1. Deadline and Reservation price. Here $V^{b}=\left\langle T^{s}, R P^{s}\right\rangle$ and $V^{s}=$ $\left\langle T^{b}, R P^{b}\right\rangle$. With this information available to both agents, the optimal strategies can be determined using backward induction as follows. Consider the case where $\left(T^{s}<T^{b}\right)$ shown in Figure 6 . The thick line denotes $b$ 's strategy and dashed lines denote $s$ 's strategies. No matter which strategy $s$ uses, it is bound to reach $R P^{s}$ by $T^{s}$ since it would have to quit if agreement is not reached by $T^{s}$. Agent $b$ can use this information to maximize its utility by never offering a price more than $R P^{s}$ prior to $T^{s}$. If $b$ gains on time, its optimal strategy, $S^{b}$, is $\left\langle I P^{b}, R P^{s}, T^{s}, B\right\rangle$ since $B$ has the property of not reaching $R P^{s}$ before $T^{s}$. On the other hand if $b$ loses on time, it tries to reach an early agreement and $S^{b}$ becomes $\left\langle I P^{b}, R P^{s}, T^{s}, C\right\rangle$. Analogously, the optimal strategy for $s, S^{s}$, becomes $\left\langle I P^{s}, R P^{s}, T^{s}, B\right\rangle$ if it gains utility with time or $\left\langle I P^{s}, R P^{s}, T^{s}, C\right\rangle$ if it loses utility with time. In the other case where $\left(T^{b}<T^{s}\right)$, $s$ will maximize its utility by never offering a price lower than $R P^{b}$ before $T^{b}$. Thus $S^{s}$ is $\left\langle I P^{s}, R P^{b}, T^{b}, B\right\rangle$ if it gains on time, and $\left\langle I P^{s}, R P^{b}, T^{b}, C\right\rangle$ if it loses on time. $S^{b}$ is $\left\langle I P^{b}, R P^{b}, T^{b}, B\right\rangle$ if it gains on time and $\left\langle I P^{b}, R P^{b}, T^{b}, C\right\rangle$ if it 
loses on time. Here again the proof of Theorem 1 can be used to show the existence of a unique equilibrium at $S^{b} \times S^{s}$. See Table I for the corresponding equilibrium outcomes.

2. Reservation price and Discounting factor. Here $V^{b}=\left\langle R P^{s}, \delta^{s}\right\rangle$ and $V^{s}=\left\{R P^{b}, \delta^{b}\right\}$. An agent's optimal strategy when $V^{a}=\left\langle R P^{\hat{a}}\right\rangle$ is independent of its opponent's discounting factor. Adding the opponent's discounting factor to $V^{a}$ therefore gives the same equilibrium strategies and outcomes as for $V^{a}=\left\langle R P^{\hat{a}}\right\rangle$.

3. Deadline and Discounting factor. As in the previous case, this gives the same equilibrium outcomes as for $V^{a}=\left\langle T^{\hat{a}}\right\rangle$.

\section{3. $V^{a}$ CONTAins THREE ELEMENTS}

Here $V^{b}=\left\langle T^{s}, R P^{s}, \delta^{s}\right\rangle$ and $V^{s}=\left\langle T^{b}, R P^{b}, \delta^{b}\right\rangle$. The optimal strategies of agents for $V^{a}=\left\langle T^{\hat{a}}, R P^{\hat{a}}\right\rangle$ do not depend on the opponent's discounting factor (see Section 3.2 for $n=2$ ). This gives the same equilibrium outcomes as for $V^{a}=\left\langle T^{\hat{a}}, R P^{\hat{a}}\right\rangle$. But $\delta^{\hat{a}}$ can be used to infer the opponent's NDF. For $(n=1)$ and $(n=2)$ we showed that a patient player uses the Boulware NDF and an impatient player uses the Conceder NDF. This makes $\mathbf{G}$ a game of complete information and both agents can pre-compute the negotiation outcome with the available information. The outcome is the same as the one obtained without $\delta^{a}$ (see Table I) but its inclusion eliminates the need for agents to go through the process of negotiation to arrive at it.

\section{Equilibrium Outcomes for Asymmetric Information Scenarios}

In the previous section the buyer and the seller had equal information about each other and they had information on the same parameter(s) about each other. This section analyses bargaining scenarios where the agents have information on different parameters about each other (i.e., they have unequal information about each other). As before, each agent has three parameters. An agent's optimal strategy is always determined on the basis of its own information state and does not depend on the opponent's information state. Put differently, agent $a$ 's optimal strategy depends solely on $I^{a}$ and is independent of $I^{\hat{a}}$. Thus changing $I^{\hat{a}}$ does not alter $a$ 's optimal strategy as long as $I^{a}$ remains the same. So agent $a$ 's optimal strategies remain the same for both symmetric and asymmetric information scenarios. However, as the combination of 
the two information states (i.e., $I^{a}$ and $I^{\hat{a}}$ ) changes, the strategy profile and, consequently, the equilibrium outcome changes.

We begin our analysis with those negotiation scenarios in which agent $a$ knows one, two or all three parameters about agent $\hat{a}$ but $\hat{a}$ has no information about $a$. Each of these situations is studied below. The equilibrium outcomes when $V^{s}=\langle\rangle$ are listed in Table II. The corresponding outcomes for $V^{b}=\langle\rangle$ can be obtained analogously.

1. Deadline. Here agent $a$ knows $\hat{a}$ 's deadline but $\hat{a}$ knows nothing about $a$. This gives rise to two scenarios $-\left(V^{b}=\left\langle T^{s}\right\rangle\right.$ and $\left.V^{s}=\langle\rangle\right)$ or $\left(V^{s}=\left\langle T^{b}\right\rangle\right.$ and $\left.V^{b}=\langle\rangle\right)$. Consider $\left(V^{b}=\left\langle T^{s}\right\rangle\right.$ and $\left.V^{s}=\langle\rangle\right)$ first. From Section 3.1 we know that the buyer's optimal strategy (determined on the basis of its own information state) is $S^{b}=$ $\left\langle I P^{b}, R P^{b}, T, B / C\right\rangle$, in which the last element is $B$ if $\delta^{b}>1$ and $C$ if $\delta^{b}<1$. As observed in the previous section, agent $a$ 's optimal strategy for $V^{a}=\langle\rangle$ is the same as for $V^{a}=\left\langle\delta^{\hat{a}}\right\rangle$. Thus the seller's optimal strategy is $S^{s}=\left\langle I P^{s}, R P^{s}, T^{s}, C\right\rangle$. The strategy profile $\left\langle I P^{b}, R P^{b}, T, B / C\right\rangle \times\left\langle I P^{s}, R P^{s}, T^{s}, C\right\rangle$ therefore forms the equilibrium. Analogously the equilibrium when $\left(V^{s}=\left\langle T^{b}\right\rangle\right.$ and $\left.V^{b}=\langle\rangle\right)$ becomes $\left\langle I P^{b}, R P^{b}, T^{b}, C\right\rangle \times\left\langle I P^{s}, R P^{s}, T, B / C\right\rangle$.

2. Reservation price. For this case $\left(V^{b}=\left\langle R P^{s}\right\rangle\right.$ and $\left.V^{s}=\langle\rangle\right)$ or $\left(V^{s}=\left\langle R P^{b}\right\rangle\right.$ and $\left.V^{b}=\langle\rangle\right)$. Again from Section 3.1, the equilibrium strategy profile for $\left(V^{b}=\left\langle R P^{s}\right\rangle\right.$ and $\left.V^{s}=\langle\rangle\right)$ is $S^{b} \times S^{s}=$ $\left\langle R P^{s}, R P^{b}, T^{b}, B / C\right\rangle \times\left\langle I P^{s}, R P^{s}, T^{s}, C\right\rangle$ and for $\left(V^{s}=\left\langle R P^{b}\right\rangle\right.$ and $\left.V^{b}=\langle\rangle\right)$ it is $\left\langle I P^{b}, R P^{b}, T^{b}, C\right\rangle \times\left\langle R P^{b}, R P^{s}, T^{s}, B / C\right\rangle$.

3. Discounting Factor. On the same lines the equilibrium for both, $\left(V^{b}=\left\langle\delta^{s}\right\rangle\right.$ and $\left.V^{s}=\langle\rangle\right)$ and $\left(V^{s}=\left\langle\delta^{b}\right\rangle\right.$ and $\left.V^{b}=\langle\rangle\right)$ is $S^{b} \times S^{s}=$ $\left\langle I P^{b}, R P^{b}, T^{b}, C\right\rangle \times\left\langle I P^{s}, R P^{s}, T^{s}, C\right\rangle$.

4. Deadline and reservation price. Here $\left(V^{b}=\left\langle T^{s}, R P^{s}\right\rangle\right.$ and $\left.V^{s}=\langle\rangle\right)$ or $\left(V^{s}=\left\langle T^{b}, R P^{b}\right\rangle\right.$ and $\left.V^{b}=\langle\rangle\right)$. For the former case, the equilibrium strategies are $S^{b} \times S^{s}=\left\langle I P^{b}, R P^{s}, T^{s}, B / C\right\rangle \times\left\langle I P^{s}, R P^{s}, T^{s}, C\right\rangle$ if $T^{s}<T^{b}$ and $\left\langle I P^{b}, R P^{b}, T^{b}, B / C\right\rangle \times\left\langle I P^{s}, R P^{s}, T^{s}, C\right\rangle$ if $T^{b}<T^{s}$. For the latter case they are $\left\langle I P^{b}, R P^{b}, T^{b}, C\right\rangle \times\left\langle I P^{s}, R P^{b}, T^{b}, B / C\right\rangle$ if $T^{b}<T^{s}$ and $\left\langle I P^{b}, R P^{b}, T^{b}, C\right\rangle \times\left\langle I P^{s}, R P^{s}, T^{s}, B / C\right\rangle$ if $T^{s}<T^{b}$.

5. Deadline, reservation price and discounting factor. An agent's optimal strategy does not depend on its opponent's discounting factor. The equilibrium for this scenario is thus the same as the previous one.

The equilibrium outcomes for the remaining negotiation scenarios (i.e., $\left.V^{s}=\left\langle T^{b}\right\rangle, V^{s}=\left\langle R P^{b}\right\rangle, V^{s}=\left\langle\delta^{b}\right\rangle, V^{s}=\left\langle T^{b}, R P^{b}\right\rangle\right)$ can be determined 
in the same way. The results for each of these scenarios are summarised in Table III to Table VI in Appendix B.

\section{Bargaining Power}

Having determined the equilibrium outcomes for symmetric and asymmetric information scenarios, we now study how the agents' information states influence their benefits from bargaining. The distribution property of a negotiation outcome relates to the issue of how the gains from trade are divided between the players. The price $\left(P_{e}\right)$ and time $\left(T_{e}\right)$ of the equilibrium agreement reflect the relationship between agents' bargaining powers. We say that an agent has more (less) bargaining power than its opponent if $\left(P_{e}, T_{e}\right)$ is more (less) in its favour than its opponent. Assume that the price-surplus is split between $b$ and $s$ in the ratio $x: y$. The agent $b$ is said to have more (less) power over price if $x>y(x<y)$. In other words, an agent's bargaining power is determined on the basis of its share of the price-surplus. Regarding the time of agreement, if $a$ prefers an early (late) agreement but $\hat{a}$ prefers a late (early) agreement and the actual time of agreement is the earlier (later) deadline, then $\hat{a}$ is said to have more (less) bargaining power than $a$ over time. For the same time preferences, if the time of agreement is $T_{0}$, then $a$ is said to have more (less) power than $\hat{a}$. Note that we do not use the agents' utility functions to determine their bargaining power since these functions can be subjective ${ }^{3}$. We now study the influence of the agents' information about their opponent's parameters on the bargaining power for symmetric information scenarios. The analysis for asymmetric information can be undertaken in the same way. These results are summarised in Table I through Table VI.

1. Influence of opponent's deadline. When agents know each others' deadline, the patient agent has equal or more power than its opponent over both price and time. This can be explained as follows. Consider first the case where agents have similar time preferences. The price-surplus is divided equally between the agents (see Section 3 ) giving them equal power over price. When both gain on time, agreement is reached at the earlier deadline and when both lose on time, agreement is reached towards the beginning of negotiation. In other words, the time of agreement is as favourable as possible to both agents, giving them equal power over time. When agents have conflicting time preferences, the entire price-surplus goes to the

\footnotetext{
${ }^{3}$ In many economic and social choice theory contexts, one unit of utility for the buyer is not equivalent to one unit of utility for the seller.
} 
patient agent and agreement is reached at the earlier deadline. This happens because although the impatient player attempts to reach an early agreement by using the Conceder NDF, its opponent's strategy delays agreement till the earlier deadline. Thus both $P_{e}$ and $T_{e}$ are in favour of the patient agent giving it more power than its opponent.

2. Influence of opponent's reservation-price. When agents know each others' reservation price, the patient agent has either more or less power than its opponent and the impatient agent has equal or less power than its opponent over price. With respect to time, the patient agent has equal or less power than its opponent and the impatient agent has equal or more power than its opponent. This can be explained as follows. Consider similar time preferences first. When both gain on time, the price-surplus goes to the agent with the longer deadline giving it more power than its opponent. When both lose on time, the price-surplus is divided equally between the agents giving them equal power over $P_{e}$. Agreement is reached at the earlier deadline when both gain on time and at the beginning of negotiation when both lose on time. Thus $T_{e}$ is as favourable as possible to both agents giving them equal power. When agents have conflicting time preferences, the price-surplus goes to the patient agent and agreement is reached towards the beginning of negotiation. This happens because the initial offers are $R P^{s}$ for $b$ and $R P^{b}$ for $s$. The time of agreement is in favour of the impatient agent. Thus the patient agent has more power over $P_{e}$ but the impatient agent has more power over $T_{e}$.

3. Influence of deadline and reservation-price. When agents know each others' deadline and reservation price, the agent with the longer deadline always has more power than its opponent over price. An agent's power over time depends on its attitude towards time. The patient agent has equal or more power than its opponent over time. This parameter combination always gives the entire price-surplus to the agent with the longer deadline giving it more power over price. Agreement is reached at the earlier deadline when at least one agent gains on time and at the beginning of negotiation when both lose on time. Thus agents have equal power in the case of like time preferences, but the patient agent gets more power in the case of conflicting time preferences.

4. Influence of opponent's discounting factor. Adding this information when there is no existing information, or when there is information on any other single parameter, or a parameter pair doesn't alter the 
equilibrium strategies or the outcome with the following exception. Adding this information to the parameter pair $\left(T^{\hat{a}}, R P^{a}\right)$ eliminates the need for negotiation between agents as the solution can be pre-computed (see Section 3 for details).

Having determined the equilibrium strategies for symmetric and asymmetric information scenarios, the next step is to find the solution properties.

\section{Properties of the Equilibrium Solutions}

A well designed negotiation mechanism should not only be simple to implement, and have stability (have strategies in equilibrium (VanDamme, 1983)) but it should also generate solutions that are unique, symmetric and efficient (Rosenschein and Zlotkin, 1994). In the proposed negotiation model, in some scenarios $T_{e}$ is $T_{0}$ (the beginning of negotiation), while in others it is $T$ (the earlier deadline). The time of agreement $\left(T_{e}\right)$ is $T_{0}$ if both the participants have a discounting factor less than one. In the remaining scenarios, it is either $T_{0}$ or $T$ depending on the agents' information state. $P_{e}$ also has only two possible values. Either the entire price-surplus goes to a single agent or both agents get an equal share of it. The following theorems show some important properties of the equilibrium outcomes in different bargaining scenarios. These properties relate to the uniqueness, symmetry and efficiency of the solution. Apart from these properties, we also study the influence of the opponent's parameters on the bargaining outcome.

Uniqueness is a desirable solution property because if the outcome is unique, then it can be identified unequivocally.

THEOREM 2. For each negotiation scenario, the proposed negotiation model has a unique equilibrium agreement.

Proof. In Section 3.1 it was shown that the solution is unique if $V^{a}=\left\langle T^{\hat{a}}\right\rangle$. Using the same proof, it can be shown that the solution is unique for the remaining scenarios. Thus for each possible symmetric and asymmetric information scenario, there is only one possible agreement.

Another desirable solution property is that of symmetry. A bargaining mechanism is said to be symmetric if it does not treat the players differently on the basis of inappropriate criteria. Exactly what constitutes inappropriate criteria depends on the specific domain. The proposed negotiation mechanism possesses the property of symmetry 
since the outcome does not depend on which player starts the process of negotiation.

THEOREM 3. In all negotiation scenarios, the bargaining outcome is independent of the identity of the first player.

Proof. As shown in Appendix B, in the equilibrium outcome for both symmetric and asymmetric information scenarios, there are two time points at which an agreement can be reached; $T_{0}$ which denotes the beginning of negotiation or $T$ which is the earlier deadline. At these time points one of the agents (either $b$ or $s$ depending on whose turn it is) offers the equilibrium solution which the other agent accepts.

An agreement is efficient if there is no wasted utility, i.e, the agreement satisfies Pareto optimality. The equilibrium solution in the proposed model is Pareto optimal in some, but not all, negotiation scenarios.

THEOREM 4. The equilibrium outcome is Pareto optimal when both agents have similar time preferences and $V^{a}$ contains $T^{\hat{a}}$ for at least one of the agents.

Proof. Let a represent the seller. Consider the asymmetric information scenarios first. The equilibrium outcomes when $V^{a}$ contains $T^{\hat{a}}$ are given in Table III and Table VI. In rows 1,2,5,6,9,10,13 and 14 of these tables the agents have similar time preferences. An agent's utility can be changed by changing the price or time of agreement or both. Consider the outcome in row 1 of Table III. The time of agreement is the earlier deadline. For this scenario, the time of agreement can only be decreased since the agent with the earlier deadline quits if agreement is not reached by T. But this decrease lowers both agents' utilities from time since $\delta^{a}>1$ for both $b$ and $s$. So both agents get the maximum possible utility from time at $T$. The price of agreement is $P$ and it can be increased or decreased. An increase in the price of agreement lowers $b$ 's utility from price and increases s's utility from price. In other words a change in time decreases the utility of both the agents while a change in price improves one agent's utility at the cost of the other agent. The outcome $(P, T)$ is therefore Pareto optimal. This argument holds good for all the remaining rows.

The same argument also holds true for the symmetric information scenarios given in rows 1,2,13 and 14 of Table I. In the same way, the property of Pareto optimality can be shown for the scenarios in which a represents the buyer. 
In the remaining negotiation scenarios, the outcome may or may not be Pareto optimal. The following theorems show the influence of the opponent's parameters on the equilibrium outcome.

THEOREM 5. Adding $\delta^{\hat{a}}$ to $V^{a}$ does not change the equilibrium outcome.

Proof. It was shown in Section 3 (for symmetric information scenarios) and Section 4 (for asymmetric information scenarios) that including $\delta^{\hat{a}}$ in $V^{a}$ does not change a's (where a is the buyer or the seller) optimal strategy. The equilibrium strategy profile and, consequently, the equilibrium outcome remains unchanged by adding $\delta^{\hat{a}}$ to $V^{a}$ or deleting $\delta^{\hat{a}}$ from $V^{a}$.

THEOREM 6. When agents know nothing about the opponent's parameters or know only the opponent's discounting factor, then the pricesurplus is split equally between the agents and agreement takes place towards the beginning of negotiation.

Proof. This is shown in Appendix B. See outcomes for $\left(V^{b}=\left\langle\delta^{s}\right\rangle\right.$ and $\left.V^{s}=\langle\rangle\right)$ and $\left(V^{b}=\left\langle\delta^{s}\right\rangle\right.$ and $\left.V^{s}=\left\langle\delta^{b}\right\rangle\right)$. The results for $\left(V^{b}=\langle\rangle\right.$ and $\left.V^{s}=\left\langle\delta^{b}\right\rangle\right)$ and $\left(V^{b}=\langle\rangle\right.$ and $\left.V^{s}=\langle\rangle\right)$ can be obtained analogously.

THEOREM 7. In all the negotiation scenarios, an impatient agent (a) is indifferent between knowing the opponent's deadline $\left(T^{\hat{a}}\right)$ and its reservation price $\left(R P^{\hat{a}}\right)$.

Proof. The impatient agent could be the seller or the buyer. Consider the seller first. The equilibrium outcomes when the seller knows the buyer's deadline and reservation price are listed in Table III and Table IV respectively. In both tables the rows 2,3,6,7,10,11,14 and 15 correspond to the scenarios where $\delta^{s}<1$. Comparing the outcomes in the two tables for rows 2 and 3 we see that the equilibrium outcomes and the resulting utilities are the same for both deadline and reservation price. This equality holds good for the equilibrium outcomes in rows 6,7,10,11,14 and 15. Thus by knowing the buyer's deadline, the seller always gets a utility that is equal to the utility it gets by knowing the buyer's reservation price irrespective of the buyer's information state.

Consider the buyer now. The entries in rows 1,2,3 and 4 in Table III, Table IV, Table $V$ and Table VI correspond to the scenarios where $b$ knows the seller's deadline. The entries in rows 5,6,7 and 8 in these four tables correspond to the scenarios where $b$ knows the seller's reservation price. $\delta^{b}<1$ in rows 2,4,6 and 8 of these tables. Consider Table III first. The equilibrium outcome in row 2 is equal to the equilibrium outcome in row 6 and the outcome in row 4 is equal to the outcome in row 8. This equality holds good between rows 2 and 6 and rows 4 and 8 of the 
remaining 3 tables. Thus by knowing the seller's deadline, the buyer always gets a utility that is equal to the utility it gets by knowing the seller's reservation price irrespective of the seller's information state. This can easily be verified for the symmteric information scenarios as well.

THEOREM 8. A patient agent (a)'s utility over time if $\left(V^{a}=\left\langle T^{\hat{a}}\right\rangle\right)$ is greater than or equal to its utility over time if $\left(V^{a}=\left\langle R P^{\hat{a}}\right\rangle\right)$ or $\left(V^{a}=\left\langle\delta^{\hat{a}}\right\rangle\right)$ irrespective of its opponent's information state $\left(V^{\hat{a}}\right)$.

Proof. Let a represent the seller. Consider first the relation between the outcomes for $\left(V^{a}=\left\langle T^{\hat{a}}\right\rangle\right)$ given in Table III and $\left(V^{a}=\left\langle R P^{\hat{a}}\right\rangle\right)$ given in Table IV. $\delta^{s}>1$ in rows 1,4,5,8,9,12,13 and 16. The time of agreement is equal in both the tables in rows 1,5 and 13, while it is greater in Table III in rows 4,8,9,12, and 16. Since $\delta^{s}>1$, s's utility if $\left(V^{a}=\left\langle T^{\hat{a}}\right\rangle\right)$ is greater than or equal to its utility if $\left(V^{a}=\left\langle R P^{\hat{a}}\right\rangle\right)$. In the same way, by comparing the entries in Table III with the entries in Table $V$ it can be seen that $U^{a}(t)$ if $\left(V^{a}=\left\langle T^{\hat{a}}\right\rangle\right)$ is greater than or equal to $U^{a}(t)$ if $\left(V^{a}=\left\langle\delta^{\hat{a}}\right\rangle\right)$.

It can easily be verified that this result holds true for the symmetric information scenarios and also if a represents the buyer.

THEOREM 9. A patient agent (a) has equal or more bargaining power over time if it knows the opponent's deadline $\left(V^{a}=\left\langle T^{\hat{a}}\right\rangle\right)$ irrespective of the opponent's information state $\left(V^{\hat{a}}\right)$.

Proof. Let a represent the seller. Table III gives the bargaining outcomes for $\left(V^{a}=\left\langle T^{\hat{a}}\right\rangle\right)$. The rows 1,4,5,8,9,12,13 and 16 represent the scenarios where $\delta^{a}>1$. In rows 1,5,9 and 13 the seller and buyer have equal power. But in rows 4,8,12 and 16 the seller has more bargaining power than the buyer. It can easily be verified that the same result holds good for symmetric information scenarios and also when a represents the buyer.

THEOREM 10. Agents have equal power over time if they have similar time preferences.

Proof. As seen in the last column of Table I, Table II, Table III, Table IV, Table $V$ and Table VI the agents have equal bargaining power in rows 1,2,5, 6, 9,10,13 and 14 that correspond to similar time preferences. 


\section{Related Work}

Initial game theoretic research typically dealt with coordination and negotiation issues by assuming that agents have complete information about each other and then giving pre-computed solutions to specific problems (Nash, 1950; Harsanyi, 1956). Also the bargainers were assumed to have no time preferences. In (Rubinstein, 1982), Rubinstein took the time preferences of bargainers into consideration in the form of their discounting factors, but again assumed complete information. A number of strategic models were later explored to explain the deadline effect on the bargaining outcome. The complete information models among these include (Fershtman and Seidmann, 1993) and (Ma and Manove, 1993). Fershtman and Seidmann model deadline effects in a multi-period sequential bargaining model in which the player who will propose in each period is chosen by a lottery. Equilibrium behaviour in this model depends on the discount factor; if it is low, agreement is reached in the first period, but if it is high enough, then the game will end in the last period with the proposer receiving all the surplus. $\mathrm{Ma}$ and Manove's model is also one of complete information but with imperfect player control over the timing of the offers. Agreements in this model tend to be made near the deadline and the division of surplus is close to an even split.

The complete information assumption is limiting because uncertainty is endemic in most realistic applications. In the latter category, information may be lacking about a variety of factors in the bargaining problem. Each player may have some private information about its own situation that is unavailable to the other players, while having only probabilistic information about the private information about other players. Following (Harsanyi and Selten, 1972), models of games of incomplete information proceed by adopting the assumption that all players start with the same probability distribution on this private information and that these priors are common knowledge. This is modelled by having the game begin with a probability distribution, known to all players. Thus players not only have priors over other players' private information, they also know what priors the other players have over their own private information. Strategic models of incomplete information thus include an extra level of detail, since they specify not only the actions and information available to the other players in the course of the game, but also their probability distributions and information prior to the start of the game. This idea has been used by a number of researchers to explore incomplete information scenarios. For instance, Rubinstein extended his complete information model (Rubinstein, 1982) to handle incomplete information in (Rubinstein, 
1985; Osborne and Rubinstein, 1994). This is an infinite horizon model that considers uncertainty over player's discounting factors. One of the players, say player 2, may be one of two types: weak (for high discounting factor) and strong (for low discounting factor). Player 1 adopts an initial belief about the identity of player 2. Player 1's preference is known to player 2. Agreement is reached in the first or second time period. The main result of the work is the existence of a unique sequential equilibrium when player 1's belief that player 2 is of type weak, is higher than a certain threshold and another unique equilibrium when this belief is lower than the threshold.

Other models of incomplete information include (Fudenberg et al., 1985; Fudenberg and Tirole, 1983; Sandholm and Vulkan, 1999; Fatima et al., 2002c). Fudenberg and Tirole (Fudenberg and Tirole, 1983) analyse an infinite horizon bargaining game by taking the players' valuations and a probability distribution over them as common knowledge. Fudenberg et al (Fudenberg et al., 1985) analyse buyer-seller infinite horizon bargaining games in which reservation prices are uncertain, but time preferences are known. Sandholm and Vulkan (Sandholm and Vulkan, 1999) consider uncertainty over agent deadlines. A common feature of all these models is that they treat the information state of agents as common knowledge. Fatima et al (Fatima et al., 2002c) address uncertainty over two parameters; deadlines and reservation prices by treating each agent's information as its private knowledge. This model was extended in (Fatima et al., 2002b; Fatima et al., 2003) to handle multiple issues. Each of these models is formulated for a different environment and the strategic behaviour of agents is studied under the chosen environment.

Our objective here is not to address the issue of uncertainty ${ }^{4}$ per se (as in the aforementioned models). Rather, our aim is to analyse the impact of knowing various pieces of information about the opponent's parameters on the negotiation outcome. To the best of our knowledge this analysis has not previously been undertaken. Moreover, none of the above mentioned models use negotiation decision functions for counter offer generation. We explore the complete range of negotiation scenarios by varying the degree of information an agent has about its opponent. Thus another difference between the existing work and ours is that while the existing models analyse the strategic behaviour of agents in specific negotiation scenarios, our work provides a comprehensive analysis of outcomes across a wide range of scenarios.

\footnotetext{
${ }^{4}$ The issue of uncertainty for our model was studied in (Fatima et al., 2002b; Fatima et al., 2002c; Fatima et al., 2003).
} 


\section{Conclusions}

This paper highlights the importance of information possessed by participants in a game. We analysed the process and outcomes of bilateral negotiation over the entire range of symmetric and asymmetric information scenarios. This was carried out by by varying the information state of agents. We determined the equilibrium for these scenarios and analysed the relative influences of the opponent's parameters on the equilibrium outcome. A number of important properties of the equilibrium outcome were also studied.

Our main conclusions are as follows. Firstly, in some negotiation scenarios agreement is reached at the earlier deadline, while in others it is reached towards the beginning of negotiation. Secondly, in some scenarios the entire price surplus goes to a single agent while in others the surplus is split equally between the two agents. Thirdly, the proposed bargaining model generates solutions that are always unique and symmetric (irrespective of the agents' information states) but not always Pareto optimal (since agents bargain with incomplete information). Finally, we showed a number of interesting properties that relate to the relative impacts of the opponent's parameters on the outcome.

We believe that the results of our analysis can be particularly useful for constructing software agents for electronic commerce for the following main reasons. Firstly, constructing software agents that optimally negotiate on behalf of real world parties that they represent is easy because our model uses simple functions for generating offers and counter offers. Secondly, our model is realistic in the sense that it incorporates time (in the form of deadlines and discounting factors) and incomplete information. Thirdly, we provide a comprehensive analysis by not only taking into account all possible relationships between agent deadlines and discounting factors but also considering all possible combinations of information states. This gives the end user a clear picture of the possible outcomes that can result given its own information state. Fourthly, the question of who will start the process of negotiation does not arise, because for each possible negotiation scenario the equilibrium outcome is unique and symmetric. Thus our analysis is important not only from the perspective of the software agent developer but also from the perspective of the end user.

In the present analysis an agent's strategy was defined in terms of the remaining time for negotiation. However, this does not always result in a fair division of the surplus, i.e., the the entire surplus goes to one of the two agents. It would therefore be interesting to explore these scenarios by defining an agent's strategy not just in terms of remaining time but also including the concessions made by the opponent in the 
previous rounds as another parameter and decide what to offer next, on the basis of both these parameters.

\section{ACKnowledgements}

This research was supported by the EPSRC under grant GR/M07052.

\section{References}

Faratin, P., C. Sierra, and N. R. Jennings: 1998, 'Negotiation decision functions for autonomous agents'. International Journal of Robotics and Autonomous Systems 24(3-4), 159-182.

Fatima, S. S., M. Wooldridge, and N. R. Jennings: 2002a, 'The influence of information on negotiation equilibrium'. In: J. Padget, O. Shehory, D. Parkes, N. Sadeh, and W. E. Walsh (eds.): Agent Mediated Electronic Commerce IV, Designing Mechanisms and Systems. pp. 180 - 193, Springer Verlag.

Fatima, S. S., M. Wooldridge, and N. R. Jennings: 2002b, 'Multi-issue negotiation under time constraints.'. In: Proceedings of the First International Conference on Autonomous Agents and Multi-Agent Systems (AAMAS-02). Bologna, Italy, pp. $143-150$.

Fatima, S. S., M. Wooldridge, and N. R. Jennings: 2002c, 'Optimal negotiation strategies for agents with incomplete information'. In: J. J. Meyer and M. Tambe (eds.): Intelligent Agents VIII. Agent Theories, Architectures and Languages. pp. 377-392, Springer Verlag.

Fatima, S. S., M. Wooldridge, and N. R. Jennings: 2003, 'An agenda based framework for multi-issue negotiation'. Artificial Intelligence Journal (to appear).

Fershtman, C. and D. J. Seidmann: 1993, 'Deadline effects and inefficient delay in bargaining with endogenous commitment'. Journal of Economic Theory 60(2), $306-321$.

Fudenberg, D., D. Levine, and J. Tirole: 1985, 'Infinite horizon models of bargaining with one sided incomplete information'. In: A. Roth (ed.): Game Theoretic Models of Bargaining. University of Cambridge Press, Cambridge.

Fudenberg, D. and J. Tirole: 1983, 'Sequential bargaining with incomplete information'. Review of Economic Studies 50, 221-247.

Harsanyi, J. and R. Selten: 1972, 'A generalized Nash solution for two-person bargaining games with incomplete information'. Management Science 18(5), 80-106.

Harsanyi, J. C.: 1956, 'Approaches to the bargaining problem before and after the theory of games'. Econometrica 24, 144-157.

Jennings, N. R., P. Faratin, A. R. Lomuscio, S. Parsons, and M. Wooldridge: 2001, 'Automated negotiation: prospects, methods and challenges'. Group Decision and Negotiation 10(2), 199-215.

Jennings, N. R., P. Faratin, T. J. Norman, P. O'Brien, B. Odgers, and J. L. Atly: 2000, 'Implementing a business process management system using adept: A realworld case study'. Int Journal of Applied Artificial Intelligence 14(5), 421-463.

Keeney, R. and H. Raiffa: 1976, Decisions with Multiple Objectives: Preferences and Value Tradeoffs. New York: John Wiley.

Kraus, S.: 2001, Startegic negotiation in multi-agent environments. The MIT Press, Cambridge, Massachusetts. 
Kreps, D. M. and R. Wilson: 1982, 'Sequential Equilibrium'. Econometrica 50, 863-894.

Lomuscio, A., M. Wooldridge, and N. R. Jennings: 2003, 'A classification scheme for negotiation in electronic commerce'. International Journal of Group Decision and Negotiation 12(1), 31-56.

Ma, C. A. and M. Manove: 1993, 'Bargaining with deadlines and imperfect player control'. Econometrica 61, 1313-1339.

Nash, J. F.: 1950, 'The bargaining problem'. Econometrica 18, 155-162.

Osborne, M. J. and A. Rubinstein: 1994, A Course in Game Theory. The MIT Press.

Pruitt, D. G.: 1981, Negotiation Behavior. Academic Press.

Raiffa, H.: 1982, The Art and Science of Negotiation. Cambridge, USA: Harvard University Press.

Rosenschein, J. S. and G. Zlotkin: 1994, Rules of Encounter. MIT Press.

Rubinstein, A.: 1982, 'Perfect equilibrium in a bargaining model'. Econometrica 50(1), 97-109.

Rubinstein, A.: 1985, 'A bargaining model with incomplete information about time preferences'. Econometrica 53, 1151-1172.

Sandholm, T.: 2000, 'Agents in electronic commerce: component technologies for automated negotiation and coalition formation.'. Autonomous Agents and MultiAgent Systems 3(1), 73-96.

Sandholm, T. and N. Vulkan: 1999, 'Bargaining with deadlines'. In: AAAI-99. Orlando, FL, pp. 44-51.

VanDamme, E.: 1983, Refinements of the Nash equilibrium concept. Berlin:SpringerVerlag.

Young, O. R.: 1975, Bargaining: Formal theories of negotiation. Urbana: University of Illinois Press.

\section{Appendix}

\section{A. A summary of notation}

\section{$b$ Buyer}

$s$ Seller

$a$ An element of the set $\{b, s\}$

$\hat{a}$ Agent $a$ 's opponent

$I P^{a}$ Initial price at which agent $a$ starts negotiation

$F P^{a}$ Final price beyond which agent $a$ does not concede

$R P^{a}$ Agent $a$ 's reservation price

$P \frac{R P^{s}+R P^{b}}{2}$ 
$Z$ The zone of agreement, i.e., a price in the interval $\left[R P^{s}, R P^{b}\right]$

$p_{b \rightarrow s}^{t}$ Price offered by $b$ to $s$ at time $t$

$B$ Boulware negotiation decision function

$C$ Conceder negotiation decision function

$L$ Linear negotiation decision function

$I^{a}$ Information state of agent $a$

$F^{a}$ Fixed part of $I^{a}$

$V^{a}$ Variable part of $I^{a}$

$T^{a}$ Agent $a$ 's deadline

$T$ The earlier deadline

$T_{0}$ The beginning of negotiation

$\delta^{a}$ Agent $a$ 's discounting factor

$U^{a}$ Agent $a$ 's utility

$S^{a}$ Agent $a$ 's optimal strategy

$P_{e}$ Equilibrium price

$T_{e}$ Time of equilibrium agreement

$G$ Indicates a discounting factor greater than 1

$L$ Indicates a discounting factor less than 1

$\dagger$ Indicates the outcome if $T^{s}<T^{b}$

$\ddagger$ Indicates the outcome if $T^{b}<T^{s}$ 


\section{B. Equilibrium outcomes for symmetric and asymmetric information scenarios}

Table I. Equilibrium outcomes and bargaining power for symmetric information scenarios.

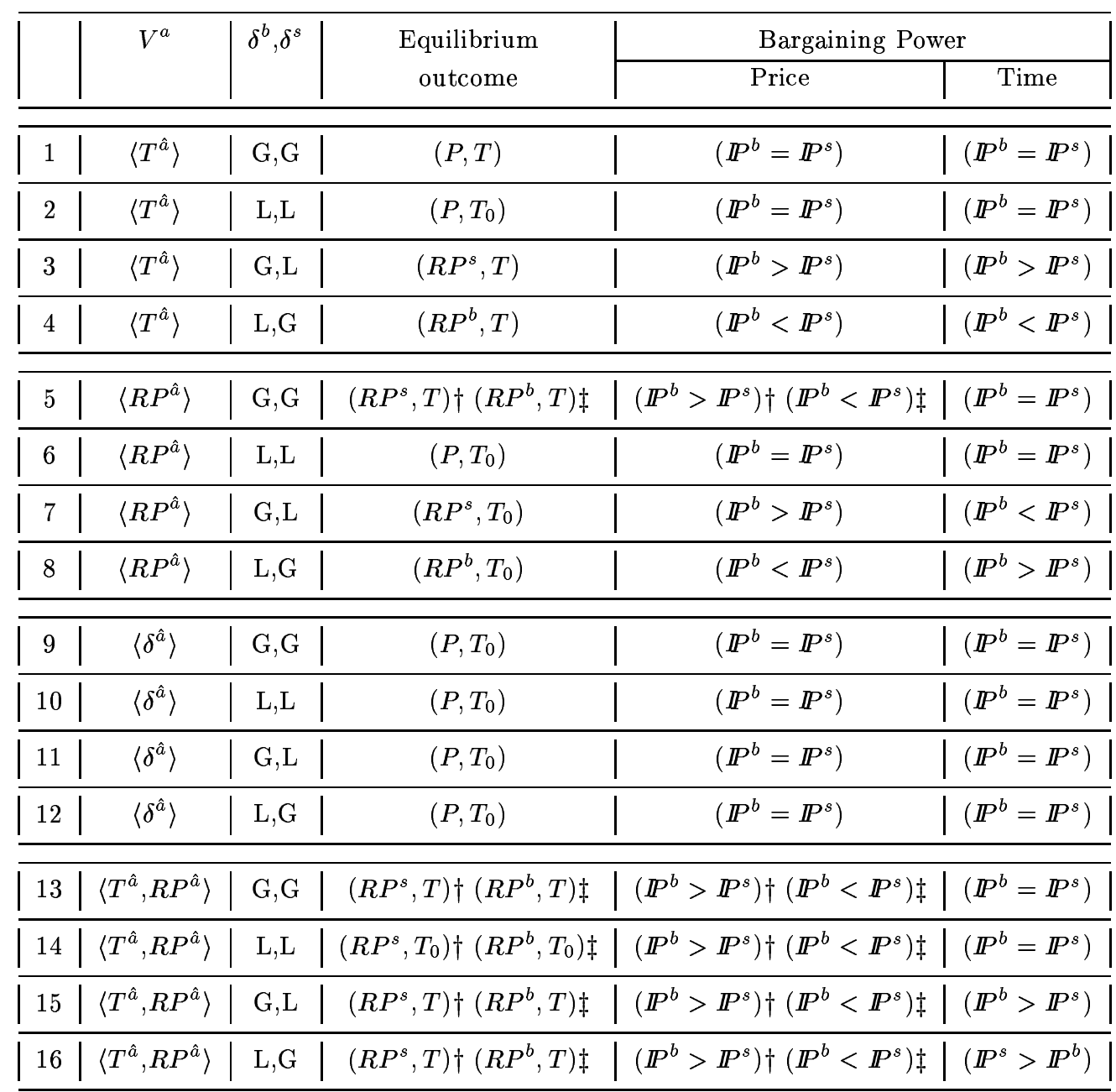


Table II. Equilibrium outcomes and bargaining power for $V^{s}=\langle\rangle$.

\begin{tabular}{|c|c|c|c|c|}
\hline \multirow[t]{2}{*}{$V^{b}$} & \multirow[t]{2}{*}{$\delta^{b}, \delta^{s}$} & \multirow{2}{*}{$\begin{array}{c}\text { Equilibrium } \\
\text { outcome }\end{array}$} & \multicolumn{2}{|c|}{ Bargaining Power } \\
\hline & & & Price & Time \\
\hline$\left\langle T^{s}\right\rangle$ & $\mathrm{G}, \mathrm{G}$ & $\left(R P^{s}, T\right)$ & $\left(\mathbb{P}^{b}>\mathbb{P}^{s}\right)$ & $\left(\mathbb{P}^{b}=\mathbb{P}^{s}\right)$ \\
\hline$\left\langle T^{s}\right\rangle$ & $\mathrm{L}, \mathrm{L}$ & $\left(P, T_{0}\right)$ & $\left(\mathbb{P}^{b}=\mathbb{P}^{s}\right)$ & $\left(\mathbb{P}^{b}=\mathbb{P}^{s}\right)$ \\
\hline$\left\langle T^{s}\right\rangle$ & $\mathrm{G}, \mathrm{L}$ & $\left(R P^{s}, T\right)$ & $\left(\mathbb{P}^{b}>\mathbb{P}^{s}\right)$ & $\left(\mathbb{P}^{b}=\mathbb{P}^{s}\right)$ \\
\hline$\left\langle T^{s}\right\rangle$ & $\mathrm{L}, \mathrm{G}$ & $\left(P, T_{0}\right)$ & $\left(\mathbb{P}^{b}=\mathbb{P}^{s}\right)$ & $\left(\mathbb{P}^{b}=\mathbb{P}^{s}\right)$ \\
\hline$\left\langle R P^{s}\right\rangle$ & $\mathrm{G}, \mathrm{G}$ & $\left(R P^{s}, T_{0}\right)$ & $\left(\mathbb{P}^{b}>\mathbb{P}^{s}\right)$ & $\left(\mathbb{P}^{b}=\mathbb{P}^{s}\right)$ \\
\hline$\left\langle R P^{s}\right\rangle$ & $\mathrm{L}, \mathrm{L}$ & $\left(P, T_{0}\right)$ & $\left(\mathbb{P}^{b}=\mathbb{P}^{s}\right)$ & $\left(\mathbb{P}^{b}=\mathbb{P}^{s}\right)$ \\
\hline$\left\langle R P^{s}\right\rangle$ & $\mathrm{G}, \mathrm{L}$ & $\left(R P^{s}, T_{0}\right)$ & $\left(\mathbb{P}^{b}>\mathbb{P}^{s}\right)$ & $\left(\mathbb{P}^{s}=\mathbb{P}^{b}\right)$ \\
\hline$\left\langle R P^{s}\right\rangle$ & $\mathrm{L}, \mathrm{G}$ & $\left(P, T_{0}\right)$ & $\left(\mathbb{P}^{b}=\mathbb{P}^{s}\right)$ & $\left(\mathbb{P}^{b}=\mathbb{P}^{s}\right)$ \\
\hline$\left\langle\delta^{s}\right\rangle$ & $\mathrm{G}, \mathrm{G}$ & $\left(P, T_{0}\right)$ & $\left(\mathbb{P}^{b}=\mathbb{P}^{s}\right)$ & $\left(\mathbb{P}^{b}=\mathbb{P}^{s}\right)$ \\
\hline$\left\langle\delta^{s}\right\rangle$ & $\mathrm{L}, \mathrm{L}$ & $\left(P, T_{0}\right)$ & $\left(\mathbb{P}^{b}=\mathbb{P}^{s}\right)$ & $\left(\mathbb{P}^{b}=\mathbb{P}^{s}\right)$ \\
\hline$\left\langle\delta^{s}\right\rangle$ & $\mathrm{G}, \mathrm{L}$ & $\left(P, T_{0}\right)$ & $\left(\mathbb{P}^{b}=\mathbb{P}^{s}\right)$ & $\left(\mathbb{P}^{b}=\mathbb{P}^{s}\right)$ \\
\hline$\left\langle\delta^{s}\right\rangle$ & $\mathrm{L}, \mathrm{G}$ & $\left(P, T_{0}\right)$ & $\left(\mathbb{P}^{b}=\mathbb{P}^{s}\right)$ & $\left(\mathbb{P}^{b}=\mathbb{P}^{s}\right)$ \\
\hline$\left\langle T^{s}, R P^{s}\right\rangle$ & $\mathrm{G}, \mathrm{G}$ & $\left(R P^{s}, T\right)$ & $\left(\mathbb{P}^{b}>\mathbb{P}^{s}\right)$ & $\left(\mathbb{P}^{b}=\mathbb{P}^{s}\right)$ \\
\hline$\left\langle T^{s}, R P^{s}\right\rangle$ & $\mathrm{L}, \mathrm{L}$ & $\left(R P^{s}, T_{0}\right) \dagger\left(P, T_{0}\right) \ddagger$ & $\left(\mathbb{P}^{b}>\mathbb{P}^{s}\right) \dagger\left(\mathbb{P}^{b}=\mathbb{P}^{s}\right) \ddagger$ & $\left(\mathbb{P}^{b}=\mathbb{P}^{s}\right)$ \\
\hline$\left\langle T^{s}, R P^{s}\right\rangle$ & $\mathrm{G}, \mathrm{L}$ & $\left(R P^{s}, T\right)$ & $\left(\mathbb{P}^{b}>\mathbb{P}^{s}\right)$ & $\left(\mathbb{P}^{b}=\mathbb{P}^{s}\right)$ \\
\hline$\left\langle T^{s}, R P^{s}\right\rangle$ & $\mathrm{L}, \mathrm{G}$ & $\left(R P^{s}, T_{0}\right) \dagger\left(P, T_{0}\right) \ddagger$ & $\left(\mathbb{P}^{b}>\mathbb{P}^{s}\right) \dagger\left(\mathbb{P}^{b}=\mathbb{P}^{s}\right) \ddagger$ & $\left(\mathbb{P}^{s}=\mathbb{P}^{b}\right)$ \\
\hline
\end{tabular}


Table III. Equilibrium outcomes and bargaining power for $V^{s}=\left\langle T^{b}\right\rangle$.

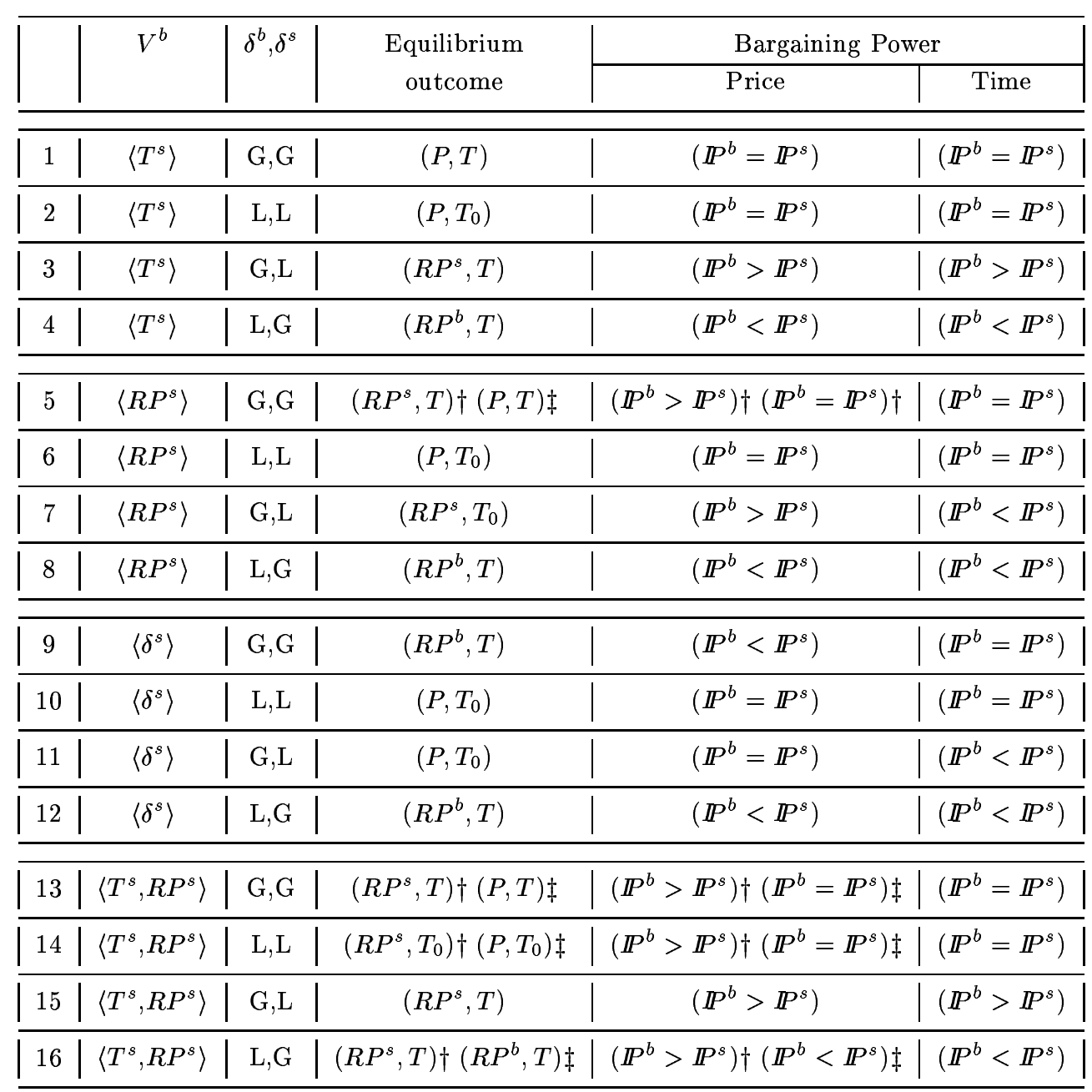


Table IV. Equilibrium outcomes and bargaining power for $V^{s}=\left\langle R P^{b}\right\rangle$.

\begin{tabular}{|c|c|c|c|c|c|}
\hline & \multirow[t]{2}{*}{$V^{b}$} & \multirow[t]{2}{*}{$\delta^{b}, \delta^{s}$} & \multirow{2}{*}{$\begin{array}{c}\text { Equilibrium } \\
\text { outcome }\end{array}$} & \multicolumn{2}{|c|}{ Bargaining Power } \\
\hline & & & & Price & Time \\
\hline 1 & $\left\langle T^{s}\right\rangle$ & $\mathrm{G}, \mathrm{G}$ & $\left(R P^{b}, T\right)$ & $\left(\mathbb{P}^{b}<\mathbb{P}^{s}\right)$ & $\left(\mathbb{P}^{b}=\mathbb{P}^{s}\right)$ \\
\hline 2 & $\left\langle T^{s}\right\rangle$ & $\mathrm{L}, \mathrm{L}$ & $\left(P, T_{0}\right)$ & $\left(\mathbb{P}^{b}=\mathbb{P}^{s}\right)$ & $\left(\mathbb{P}^{b}=\mathbb{P}^{s}\right)$ \\
\hline 3 & $\left\langle T^{s}\right\rangle$ & $\mathrm{G}, \mathrm{L}$ & $\left(R P^{s}, T\right)$ & $\left(\mathbb{P}^{b}>\mathbb{P}^{s}\right)$ & $\left(\mathbb{P}^{b}>\mathbb{P}^{s}\right)$ \\
\hline 4 & $\left\langle T^{s}\right\rangle$ & $\mathrm{L}, \mathrm{G}$ & $\left(R P^{b}, T_{0}\right)$ & $\left(\mathbb{P}^{b}<\mathbb{P}^{s}\right)$ & $\left(\mathbb{P}^{b}>\mathbb{P}^{s}\right)$ \\
\hline 5 & $\left\langle R P^{s}\right\rangle$ & $\mathrm{G}, \mathrm{G}$ & $\left(R P^{s}, T\right) \dagger\left(R P^{b}, T\right) \ddagger$ & $\left(\mathbb{P}^{b}>\mathbb{P}^{s}\right) \dagger\left(\mathbb{P}^{b}<\mathbb{P}^{s}\right) \ddagger$ & $\left(\mathbb{P}^{b}=\mathbb{P}^{s}\right)$ \\
\hline 6 & $\left\langle R P^{s}\right\rangle$ & $\mathrm{L}, \mathrm{L}$ & $\left(P, T_{0}\right)$ & $\left(\mathbb{P}^{b}=\mathbb{P}^{s}\right)$ & $\left(\mathbb{P}^{b}=\mathbb{P}^{s}\right)$ \\
\hline 7 & $\left\langle R P^{s}\right\rangle$ & $\mathrm{G}, \mathrm{L}$ & $\left(R P^{s}, T_{0}\right)$ & $\left(\mathbb{P}^{b}>\mathbb{P}^{s}\right)$ & $\left(\mathbb{P}^{b}<\mathbb{P}^{s}\right)$ \\
\hline 8 & $\left\langle R P^{s}\right\rangle$ & $\mathrm{L}, \mathrm{G}$ & $\left(R P^{b}, T_{0}\right)$ & $\left(\mathbb{P}^{b}<\mathbb{P}^{s}\right)$ & $\left(\mathbb{P}^{b}>\mathbb{P}^{s}\right)$ \\
\hline 9 & $\left\langle\delta^{s}\right\rangle$ & $\mathrm{G}, \mathrm{G}$ & $\left(R P^{b}, T_{0}\right)$ & $\left(\mathbb{P}^{b}<\mathbb{P}^{s}\right)$ & $\left(\mathbb{P}^{b}=\mathbb{P}^{s}\right)$ \\
\hline & $\left\langle\delta^{s}\right\rangle$ & $\mathrm{L}, \mathrm{L}$ & $\left(P, T_{0}\right)$ & $\left(\mathbb{P}^{b}=\mathbb{P}^{s}\right)$ & $\left(\mathbb{P}^{b}=\mathbb{P}^{s}\right)$ \\
\hline & $\left\langle\delta^{s}\right\rangle$ & $\mathrm{G}, \mathrm{L}$ & $\left(P, T_{0}\right)$ & $\left(\mathbb{P}^{b}=\mathbb{P}^{s}\right)$ & $\left(\mathbb{P}^{b}<\mathbb{P}^{s}\right)$ \\
\hline & $\left\langle\delta^{s}\right\rangle$ & $\mathrm{L}, \mathrm{G}$ & $\left(R P^{b}, T_{0}\right)$ & $\left(\mathbb{P}^{b}<\mathbb{P}^{s}\right)$ & $\left(\mathbb{P}^{b}>\mathbb{P}^{s}\right)$ \\
\hline 13 & $\left\langle T^{s}, R P^{s}\right\rangle$ & $\mathrm{G}, \mathrm{G}$ & $\left(R P^{s}, T\right) \dagger\left(R P^{b}, T\right) \ddagger$ & $\left(\mathbb{P}^{b}>\mathbb{P}^{s}\right) \dagger\left(\mathbb{P}^{b}<\mathbb{P}^{s}\right) \ddagger$ & $\left(\mathbb{P}^{b}=\mathbb{P}^{s}\right)$ \\
\hline & $\left\langle T^{s}, R P^{s}\right\rangle$ & $\mathrm{L}, \mathrm{L}$ & $\left(R P^{s}, T_{0}\right) \dagger\left(P, T_{0}\right) \dagger$ & $\left(\mathbb{P}^{b}>\mathbb{P}^{s}\right) \dagger\left(\mathbb{P}^{b}=\mathbb{P}^{s}\right) \ddagger$ & $\left(\mathbb{P}^{b}=\mathbb{P}^{s}\right)$ \\
\hline & $\left\langle T^{s}, R P^{s}\right\rangle$ & $\mathrm{G}, \mathrm{L}$ & $\left(R P^{s}, T\right)$ & $\left(\mathbb{P}^{b}>\mathbb{P}^{s}\right)$ & $\left(\mathbb{P}^{b}>\mathbb{P}^{s}\right)$ \\
\hline 16 & $\left\langle T^{s}, R P^{s}\right\rangle$ & $\mathrm{L}, \mathrm{G}$ & $\left(R P^{s}, T\right) \dagger\left(R P^{b}, T_{0}\right) \ddagger$ & $\left(\mathbb{P}^{b}>\mathbb{P}^{s}\right) \dagger\left(\mathbb{P}^{b}<\mathbb{P}^{s}\right) \ddagger$ & $\left(\mathbb{P}^{b}<\mathbb{P}^{s}\right) \dagger\left(\mathbb{P}^{b}>\mathbb{P}^{s}\right) \ddagger$ \\
\hline
\end{tabular}


Table V. Equilibrium outcomes and bargaining power for $V^{s}=\left\langle\delta^{b}\right\rangle$.

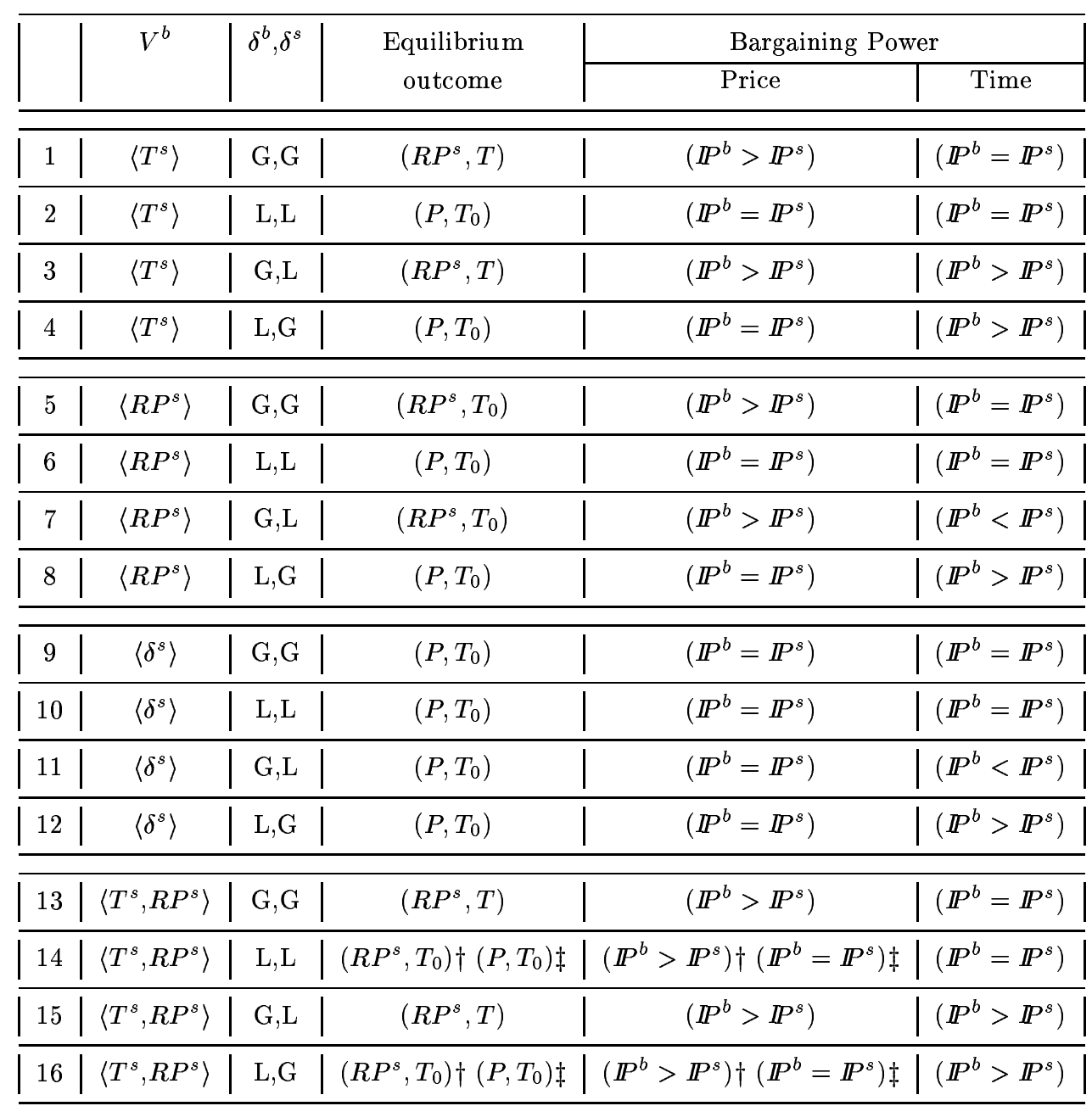


Table VI. Equilibrium outcomes and bargaining power for $V^{s}=\left\langle T^{b}, R P^{b}\right\rangle$.

\begin{tabular}{|c|c|c|c|c|c|}
\hline & \multirow[t]{2}{*}{$V^{b}$} & \multirow[t]{2}{*}{$\delta^{b}, \delta^{s}$} & \multirow{2}{*}{$\begin{array}{c}\text { Equilibrium } \\
\text { outcome }\end{array}$} & \multicolumn{2}{|c|}{ Bargaining Power } \\
\hline & & & & Price & Time \\
\hline 1 & $\left\langle T^{s}\right\rangle$ & $\mathrm{G}, \mathrm{G}$ & $(P, T) \dagger\left(R P^{b}, T\right) \ddagger$ & $\left(\mathbb{P}^{b}=\mathbb{P}^{s}\right) \dagger\left(\mathbb{P}^{b}<\mathbb{P}^{s}\right) \ddagger$ & $\left(\mathbb{P}^{b}=\mathbb{P}^{s}\right)$ \\
\hline 2 & $\left\langle T^{s}\right\rangle$ & $\mathrm{L}, \mathrm{L}$ & $\left(P, T_{0}\right) \dagger\left(R P^{b}, T_{0}\right) \ddagger$ & $\left(\mathbb{P}^{b}=\mathbb{P}^{s}\right) \dagger\left(\mathbb{P}^{b}<\mathbb{P}^{s}\right) \ddagger$ & $\left(\mathbb{P}^{b}=\mathbb{P}^{s}\right)$ \\
\hline 3 & $\left\langle T^{s}\right\rangle$ & $\mathrm{G}, \mathrm{L}$ & $\left(R P^{s}, T\right) \dagger\left(R P^{b}, T\right) \ddagger$ & $\left(\mathbb{P}^{b}>\mathbb{P}^{s}\right) \dagger\left(\mathbb{P}^{b}<\mathbb{P}^{s}\right) \ddagger$ & $\left(\mathbb{P}^{b}>\mathbb{P}^{s}\right)$ \\
\hline 4 & $\left\langle T^{s}\right\rangle$ & $\mathrm{L}, \mathrm{G}$ & $\left(R P^{b}, T\right)$ & $\left(\mathbb{P}^{b}<\mathbb{P}^{s}\right)$ & $\left(\mathbb{P}^{b}<\mathbb{P}^{s}\right)$ \\
\hline 5 & $\left\langle R P^{s}\right\rangle$ & $\mathrm{G}, \mathrm{G}$ & $\left(R P^{s}, T\right) \dagger\left(R P^{b}, T\right) \ddagger$ & $\left(\mathbb{P}^{b}>\mathbb{P}^{s}\right) \dagger\left(\mathbb{P}^{b}<\mathbb{P}^{s}\right) \ddagger$ & $\left(\mathbb{P}^{b}=\mathbb{P}^{s}\right)$ \\
\hline 6 & $\left\langle R P^{s}\right\rangle$ & $\mathrm{L}, \mathrm{L}$ & $\left(P, T_{0}\right) \dagger\left(R P^{b}, T_{0}\right) \ddagger$ & $\left(\mathbb{P}^{b}=\mathbb{P}^{s}\right) \dagger\left(\mathbb{P}^{b}<\mathbb{P}^{s}\right) \ddagger$ & $\left(\mathbb{P}^{b}=\mathbb{P}^{s}\right)$ \\
\hline 7 & $\left\langle R P^{s}\right\rangle$ & $\mathrm{G}, \mathrm{L}$ & $\left(R P^{s}, T_{0}\right) \dagger\left(R P^{b}, T\right) \ddagger$ & $\left(\mathbb{P}^{b}>\mathbb{P}^{s}\right) \dagger\left(\mathbb{P}^{b}<\mathbb{P}^{s}\right) \ddagger$ & $\left(\mathbb{P}^{b}<\mathbb{P}^{s}\right) \dagger\left(\mathbb{P}^{b}>\mathbb{P}^{s}\right) \ddagger$ \\
\hline 8 & $\left\langle R P^{s}\right\rangle$ & $\mathrm{L}, \mathrm{G}$ & $\left(R P^{b}, T\right)$ & $\left(\mathbb{P}^{b}<\mathbb{P}^{s}\right)$ & $\left(\mathbb{P}^{b}<\mathbb{P}^{s}\right)$ \\
\hline 9 & $\left\langle\delta^{s}\right\rangle$ & $\mathrm{G}, \mathrm{G}$ & $\left(R P^{b}, T\right)$ & $\left(\mathbb{P}^{b}<\mathbb{P}^{s}\right)$ & $\left(\mathbb{P}^{b}=\mathbb{P}^{s}\right)$ \\
\hline 10 & $\left\langle\delta^{s}\right\rangle$ & $\mathrm{L}, \mathrm{L}$ & $\left(P, T_{0}\right) \dagger\left(R P^{b}, T_{0}\right) \ddagger$ & $\left(\mathbb{P}^{b}=\mathbb{P}^{s}\right) \dagger\left(\mathbb{P}^{b}<\mathbb{P}^{s}\right) \ddagger$ & $\left(\mathbb{P}^{b}=\mathbb{P}^{s}\right)$ \\
\hline 11 & $\left\langle\delta^{s}\right\rangle$ & $\mathrm{G}, \mathrm{L}$ & $\left(P, T_{0}\right) \dagger\left(R P^{b}, T_{0}\right) \ddagger$ & $\left(\mathbb{P}^{b}=\mathbb{P}^{s}\right) \dagger\left(\mathbb{P}^{b}<\mathbb{P}^{s}\right) \ddagger$ & $\left(\mathbb{P}^{b}<\mathbb{P}^{s}\right)$ \\
\hline 12 & $\left\langle\delta^{s}\right\rangle$ & $\mathrm{L}, \mathrm{G}$ & $\left(R P^{b}, T\right)$ & $\left(\mathbb{P}^{b}<\mathbb{P}^{s}\right)$ & $\left(\mathbb{P}^{b}<\mathbb{P}^{s}\right)$ \\
\hline 13 & $\left\langle T^{s}, R P^{s}\right\rangle$ & $\mathrm{G}, \mathrm{G}$ & $\left(R P^{s}, T\right) \dagger\left(R P^{b}, T\right) \ddagger$ & $\left(\mathbb{P}^{b}>\mathbb{P}^{s}\right) \dagger\left(\mathbb{P}^{b}<\mathbb{P}^{s}\right) \ddagger$ & $\left(\mathbb{P}^{b}=\mathbb{P}^{s}\right)$ \\
\hline & $\left\langle T^{s}, R P^{s}\right\rangle$ & $\mathrm{L}, \mathrm{L}$ & $\left(R P^{s}, T_{0}\right) \dagger\left(R P^{b}, T_{0}\right) \ddagger$ & $\left(\mathbb{P}^{b}>\mathbb{P}^{s}\right) \dagger\left(\mathbb{P}^{b}<\mathbb{P}^{s}\right) \ddagger$ & $\left(\mathbb{P}^{b}=\mathbb{P}^{s}\right)$ \\
\hline & $\left\langle T^{s}, R P^{s}\right\rangle$ & $\mathrm{G}, \mathrm{L}$ & $\left(R P^{s}, T\right) \dagger\left(R P^{b}, T\right) \ddagger$ & $\left(\mathbb{P}^{b}>\mathbb{P}^{s}\right) \dagger\left(\mathbb{P}^{b}<\mathbb{P}^{s}\right) \ddagger$ & $\left(\mathbb{P}^{b}>\mathbb{P}^{s}\right)$ \\
\hline 16 & $\left\langle T^{s}, R P^{s}\right\rangle$ & $\mathrm{L}, \mathrm{G}$ & $\left(R P^{s}, T\right) \dagger\left(R P^{b}, T\right) \ddagger$ & $\left(\mathbb{P}^{b}>\mathbb{P}^{s}\right) \dagger\left(\mathbb{P}^{b}<\mathbb{P}^{s}\right) \ddagger$ & $\left(\mathbb{P}^{s}<\mathbb{P}^{b}\right)$ \\
\hline
\end{tabular}


p.tex; $20 / 08 / 2003 ; 10: 34 ;$ p.34 